\title{
Interdomain Identity-Based Key Agreement Schemes
}

\author{
Chun-I Fan, ${ }^{1}$ Yi-Hui Lin, ${ }^{2}$ Tuan-Hung Hsu, ${ }^{1}$ and Ruei-Hau Hsu ${ }^{3}$ \\ ${ }^{1}$ Department of Computer Science and Engineering, National Sun Yat-sen University, Kaohsiung 804, Taiwan \\ ${ }^{2}$ Institute of Information Science, Academia Sinica, Taipei 115, Taiwan \\ ${ }^{3}$ College of Computer Science, National Chiao Tung University, Hsinchu 300, Taiwan
}

Correspondence should be addressed to Chun-I Fan; cifan@faculty.nsysu.edu.tw

Received 15 April 2014; Accepted 11 August 2014; Published 30 November 2014

Academic Editor: Tadeusz Kaczorek

Copyright (C) 2014 Chun-I Fan et al. This is an open access article distributed under the Creative Commons Attribution License, which permits unrestricted use, distribution, and reproduction in any medium, provided the original work is properly cited.

\begin{abstract}
In order to simplify key management, two-party and three-party key agreement schemes based on user identities have been proposed recently. Multiparty (including more than three parties) key agreement protocols, which also are called conference key schemes, can be applied to distributed systems and wireless environments, such as ad hoc networks, for the purpose of multiparty secure communication. However, it is hard to extend two- or three-party schemes to multiparty ones with the guarantee of efficiency and security. In addition to the above two properties, interdomain environments should also be considered in key agreement systems due to diversified network domains. However, only few identity-based multiparty conference key agreement schemes for single domain environments and none for interdomain environments were proposed in the literature and they did not satisfy all of the security attributes such as forward secrecy and withstanding impersonation. In this paper, we will propose a novel efficient single domain identity-based multiparty conference key scheme and extend it to an interdomain one. Finally, we prove that the proposed schemes satisfy the required security attributes via formal methods.
\end{abstract}

\section{Introduction}

The technique of key agreement allows two or more parties to exchange information and negotiate a common session key. The first key exchange scheme was proposed by Diffie and Hellman in 1976 [1] where two parties can exchange public information and then compute a common key by their private keys and received information. However, the basic DiffieHellman protocol lacks mutual authentication between two parties such that the man-in-the-middle attack is valid in this scheme. Many researchers modified Diffie-Hellman protocol to ensure mutual authentication between two parties, which are called authenticated key agreement (AKA) protocols. Lots of varieties of Diffie-Hellman protocol have been proposed and several different kinds of key agreement mechanisms have been shown in [2]. Up to now, Diffie-Hellman key exchange protocol is still an important basis for most key agreement protocols.

In 1984, Shamir proposed an identity-based cryptosystem [3], where the public key of each user is her/his public identity information, and there exists a private key generator (PKG), a key generation center (KGC), or a Trusted Authority (TA) which is trusted by all users. PKG, KGC, or TA, which will be called TA below, can produce each user's private key according to her/his public key. In almost all of the identitybased key agreement schemes, TA provides the private/public key generation services for users. When a user registers with TA, the user's public information like ID or email address will be her/his public key and TA gives the user the private key corresponding to her/his public key.

Pairing is a tool which is initially applied to cryptography to convert the Discrete Logarithm problem in elliptic curves to that in finite fields, and it can be derived from bilinear pairing, namely, Weil pairing [4] or Tate pairing [5]. First, Joux [6] used pairing to construct the first 3-party key agreement protocol based on a certificate system in 2000 and his scheme. Later, researchers found that pairing is suitable for the implementation of identity-based cryptosystems. Smart [7] proposed a two-party identity-based authenticated key agreement scheme in 2002. Boneh and Franklin [4] proposed an identity-based encryption scheme based on Weil pairing in 2003. Afterwards, pairing has become 
an important mathematic foundation of cryptography. There are many identity-based key agreement schemes, which have been proposed in the literature [7-11], based on pairings.

A conference key agreement scheme is a variety of a multiparty key agreement or group key agreement scheme, but it is different from conference key distribution scheme. In a conference key distribution scheme, a session conference chair decides the conference key and then broadcasts it to every member in this session conference. In particular, in a conference key agreement scheme, we must guarantee that the protocol satisfies the following three properties.

(1) Each conference key is negotiated by all session members.

(2) Every session member can compute the conference key via the same algorithm.

(3) No session member can predict or preselect the conference key.

The first formal security analysis in an identity-based two-party key agreement scheme was introduced by Chen and Kudla [9] and they improved the first identity-based key agreement scheme based on pairings [7]. Chen and Kudla proved that their protocol is secure on the security model of Bellare and Rogaway [12]. Later, Al-Riyami and Paterson also proposed four kinds of tripartite authenticated key agreement protocols by improving Joux's scheme [13], and they showed that their scheme is secure. Unfortunately, Shim and Woo [14] pointed out that their scheme has some weaknesses. Furthermore, there are several conference key agreement schemes based on bilinear pairing which have been proposed in the literature [15-19], but they are all insecure, where their security weaknesses will be shown in Section 3 of the paper.

Section 4 will present two new hard problems, the $n$ Linear Diffie-Hellman ( $n$-LDH) problem and the Decisional $n$-Linear Diffie-Hellman (n-DLDH) problem, on which our key agreement schemes are based.

In Section 5, we will propose a novel efficient identitybased conference key agreement scheme by combining the concepts of $[16,19]$. In addition to a single TA, we also discuss how the users, who have registered with distinct TAs, negotiate a common conference key. Moreover, in order to formally demonstrate the security of our proposed schemes, we adopt the random oracle method, which was proposed by Bellare and Rogaway [12], to prove the security of our schemes under some well-known assumptions. We will define several security attributes in the third part of Section 2 and formally prove the security of our schemes in Section 6. Finally, we also provide performance comparison to demonstrate that our proposed schemes are more efficient than others.

Our contributions are summarized as follows.

(1) We find some security flaws in the schemes of [15-19].

(2) We introduce two new hard problems.

(3) We propose interdomain identity-based conference key agreement schemes.

(4) We formally prove that our schemes completely satisfy all of the security attributes.

\section{Preliminaries}

In this section, we review the concept of pairing which includes definitions, computationally hard problems, and security attributes of key agreement based on pairings.

2.1. Pairing. Pairing [20] in an elliptic curve cryptosystem is a function which maps a pair of elliptic curve points to an element of a multiplicative group in a finite field. It has been applied to key agreement, signatures, broadcast encryption, and identity-based encryption widely. In the following, we will review the definitions and properties of pairings.

2.1.1. Bilinear Pairing. We briefly describe the concept of bilinear pairing [20]. Let $\left(\mathbb{G}_{1},+\right)$ and $\left(\mathbb{G}_{2},+\right)$ be abelian groups written in additive notation with prime order $q$ and identity elements $O_{1}$ and $O_{2}$, respectively, such that $q P=O_{1}$ and $q Q=O_{2}$, where $\forall P \in \mathbb{G}_{1}$ and $\forall Q \in \mathbb{G}_{2}$. Suppose that $\left(\mathbb{G}_{T}, *\right)$ is a cyclic group of order $q$ written in multiplicative notation with identity element $1_{T}$. Now we have the groups $\left(\mathbb{G}_{1},+\right)$, $\left(\mathbb{G}_{2},+\right)$, and $\left(\mathbb{G}_{T}, *\right)$. The mapping function is

$$
e: \mathbb{G}_{1} \times \mathbb{G}_{2} \longrightarrow \mathbb{G}_{T}
$$

Typically, $\mathbb{G}_{1}$ and $\mathbb{G}_{2}$ are subgroups of the points on an elliptic curve over a finite field and $\mathbb{G}_{T}$ is a subgroup of a multiplicative group over a finite field.

In addition, the following additional properties must be satisfied:

(i) bilinearity

$$
\begin{aligned}
& \forall P, P^{\prime} \in \mathbb{G}_{1}, Q, Q^{\prime} \in \mathbb{G}_{2}, \\
& e\left(P+P^{\prime}, Q\right)=e(P, Q) \cdot e\left(P^{\prime}, Q\right) \text { and } e\left(P, Q+Q^{\prime}\right)= \\
& e(P, Q) \cdot e\left(P, Q^{\prime}\right), \\
& e(a P, Q)=e(P, Q)^{a}=e(P, a Q) \text { for all } a \in \mathbb{Z}_{q}^{*} ;
\end{aligned}
$$

(ii) nondegeneracy

$$
\begin{aligned}
& \forall P \in \mathbb{G}_{1} \text {, with } P \neq O_{1}, \exists Q \in \mathbb{G}_{2} \text { such that } \\
& e(P, Q) \neq 1_{T}, \\
& \forall Q \in \mathbb{G}_{2} \text {, with } Q \neq O_{2}, \exists P \in \mathbb{G}_{1} \text { such that } \\
& e(P, Q) \neq 1_{T}, \\
& \forall P \in \mathbb{G}_{1} \text { and } \forall Q \in \mathbb{G}_{2}, e\left(P, O_{2}\right)=e\left(O_{1}, Q\right)= \\
& 1_{T} ;
\end{aligned}
$$

(iii) computability

if $P \in \mathbb{G}_{1}$ and $Q \in \mathbb{G}_{2}$, there exists an efficient algorithm which can compute $e(P, Q)$ in polynomial time.

The schemes in Section 3 use symmetric bilinear pairing, so they set $\mathbb{G}_{1}=\mathbb{G}_{2}$. In order to make the following decisional problems remain hard, we set $\mathbb{G}_{1} \neq \mathbb{G}_{2}$ and there is no polynomial-time computable isomorphism $\phi: \mathbb{G}_{1} \rightarrow \mathbb{G}_{2}$, such that $\phi(P)=Q$, where $P$ is a generator of $\mathbb{G}_{1}$ and $Q$ is a generator of $\mathbb{G}_{2}$. 


\subsection{Hard Problems}

(1) The Discrete Logarithm (DL) problem: given $P, P^{\prime} \in \mathbb{G}_{1}$, find an integer $x \in \mathbb{Z}_{q}^{*}$ such that $P^{\prime}=x P$.

(2) The Computational Diffie-Hellman (CDH) problem: for $x, y \in_{R} \mathbb{Z}_{q}^{*}$, given $(P, x P, y P) \in \mathbb{G}_{1}^{3}$, compute $x y P$.

(3) The Decisional Diffie-Hellman (DDH) problem:

for $x, y \in_{R} \mathbb{Z}_{q}^{*}$, given $(P, x P, y P, z P) \in \mathbb{G}_{1}^{4}$ where $z=$ $x y(\bmod q)$ or $z \in_{R} \mathbb{Z}_{q}^{*}$ is decided by flipping a coin. Output "Yes" if $z=x y(\bmod q)$; otherwise output "No".

(4) The Divisible Computational Diffie-Hellman (DCDH) problem [21]: for $x, y \in_{R} \mathbb{Z}_{q}^{*}$, given $(P, x P, y P) \in \mathbb{G}_{1}^{3}$, compute $x y^{-1} P$.

(5) The Decisional Linear Diffie-Hellman (DLDH) problem in $\mathbb{G}_{1}[22,23]$ : for $x_{1}, x_{2}, x_{3}, x_{4} \in_{R} \mathbb{Z}_{q}^{*}$, given $\left(P, x_{1} P, x_{2} P, x_{1} x_{3} P, x_{2} x_{4} P, Z\right) \in \mathbb{G}_{1}^{6}$, where $Z=\left(x_{3}+\right.$ $\left.x_{4}\right) P$ or $Z \epsilon_{R} \mathbb{G}_{1}$ is decided by flipping a coin. Output "Yes" if $Z=\left(x_{3}+x_{4}\right) P$; otherwise output "No".

This hard problem was first proposed by Boneh et al. [22] in 2004 and then Boyen and Waters [23] extended it to asymmetric bilinear groups in 2006.

(6) The co-Bilinear Diffe-Hellman (co-BDH) problem [4]: given $\left(P_{1}, a P_{1}, b P_{1}\right) \in \mathbb{G}_{1}^{3}$ and $\left(P_{2}, a P_{2}, c P_{2}\right) \in$ $\mathbb{G}_{2}^{3}$ in asymmetric bilinear map groups $\left(\mathbb{G}_{1}, \mathbb{G}_{2}, \mathbb{G}_{T}\right)$, compute $e\left(P_{1}, P_{2}\right)^{a b c} \in \mathbb{G}_{T}$.

We propose the variant-CDH problem and extend the DLDH problem to the $n-\mathrm{LDH}$ and $n-\mathrm{DLDH}$ problems. We will prove that they are also hard in Section 4.

(7) The Variant Computational Diffie-Hellman (variant$\mathrm{CDH})$ problem: given $\left(P_{1}, a P_{1}, b P_{1}\right) \in \mathbb{G}_{1}^{3}$ and $\left(P_{2}, a P_{2}\right) \in \mathbb{G}_{2}^{2}$, compute $a b P_{1}$.

(8) The n-Linear Diffie-Hellman ( $n$-LDH) problem: given $P, x_{i} P^{\prime}$ 's, and $x_{i} x_{n+j} P^{\prime}$ 's $\in \mathbb{G}_{1}$ for all $i, j$ with $1 \leq i, j \leq n$, $i \neq j$, and $n \geq 2$, compute $\left(x_{n+1}+x_{n+2}+\cdots+x_{2 n}\right) P$.

(9) The Decisional n-Linear Diffie-Hellman (n-DLDH) problem: given $Z, P, x_{i} P^{\prime}$ 's, and $x_{i} x_{n+j} P$ 's $\in \mathbb{G}_{1}$ for all $i$, $j$ with $1 \leq i, j \leq n, i \neq j$, and $n \geq 2$, where $Z=\left(x_{n+1}+\right.$ $\left.x_{n+2}+\cdots+x_{2 n}\right) P$ or $Z \epsilon_{R} \mathbb{G}_{1}$ is decided by flipping a coin. Output "Yes" if $Z=\left(x_{n+1}+x_{n+2}+\cdots+x_{2 n}\right) P$; otherwise output "No".

2.3. Security Attributes. There are some security definitions in the identity-based key agreement schemes based on pairing $[13,14]$. We describe them as follows.

Known Session Key Security. A key agreement protocol should produce a unique common secret key, which is called a session key, for every session. The protocol should still achieve this goal when an adversary has learned all of the other session keys.
(Perfect) Forward Secrecy. Forward secrecy is that any adversary cannot derive previous session keys from compromised long-term private keys of one or more parties. Partial forward secrecy is that one or more (not all) parties' long-term private keys are corrupted but any adversary cannot get any previous session keys which were established by these parties. Perfect forward secrecy means that any adversaries cannot derive previous session keys even though they have obtained the long-term private keys of all parties. In ID-based systems, perfect forward secrecy implies that TA's and all users' long-term private keys are corrupted but any previous session key established by the registered users cannot be derived by adversaries. We also call it TA forward secrecy.

Key-Compromise Impersonation. A protocol can resist keycompromise impersonation if an adversary cannot impersonate some users even though the other users' long-term private keys were disclosed.

Man-in-the-middle attack is a special case of keycompromise impersonation in ID-based systems. If an adversary intercepts messages, retransmits them, and then communicates with users without being detected in the key agreement protocol, we say that he succeeds in impersonation.

Withstanding key-compromise impersonation also covers unknown key-share resilience. It is the basic security attribute for key agreement scheme. Some users cannot have a key agreement with the other users without the knowledge of them. If some users cannot impersonate the others, they cannot run the key agreement scheme for them.

Key Control. It should be impossible for any participant (or an adversary) to preselect a value as a session key or predict the value of the session key.

\section{Security Problems in the Previous Schemes}

In the section, we briefly introduce security weaknesses on the schemes [15-19, 24-26]. The details of the security problems in these schemes are in the Appendices.

Shi et al. [19] proposed an ID-based authenticated group key agreement protocol in 2005. The design of the protocol is efficient because it only takes one round to finish a group key agreement and it needs no exponentiation computation besides a pairing computation. We find that the protocol does not resist key-compromise impersonation since the users do not verify the messages with one another in the protocol. Moreover, it only achieves partial forward secrecy.

Du et al. [15] proposed an ID-based authenticated group key agreement protocol in 2003 and improved it in the same year. Both of them does not achieve perfect forward secrecy. Although they embed a signature scheme to verify the messages, both of the protocols still suffer from keycompromise impersonation found by Zhang and Chen [29]. In the attack of [29], the adversaries collect the messages of the user in the previous session and replay them after modifying the messages. Zhang and Chen [30] also attacked Choi et al. [24] with the same method in 2004. The protocol 
of Zhang et al. [18] in 2005 has the same security problem as Du's since they embed the same signature scheme in the protocol.

Kim et al. [17] aims to design a one-round key agreement protocol. But we find that the protocol cannot even achieve known session key security. Anyone can compute the session key through collecting the broadcasting messages.

Zhou et al. [26] proposed two schemes, one is one-round and the other is two-round. We find that both of them cannot withstand key-compromise impersonation. For the first scheme, the other users can collide to impersonate the user $U_{i}$. For the second one, the user $U_{1}$ can impersonate any other user he wants. We also find that the protocol of Yao et al. [25] is not immune to key-compromise impersonation, either. A user can impersonate another by rebroadcasting the messages. The work of [31] improved the flaw but did not provide any formal proofs. Yuan et al. [27] improved it with formal proofs.

\section{Three New Hard Problems}

We formally prove our proposed problems, the Variant Computational Diffie-Hellman problem, the $n$-Linear DiffieHellman problem, and the Decisional $n$-Linear DiffieHellman problem, being hard by using problem reduction and generic model, respectively.

\subsection{The Variant Computational Diffie-Hellman (Variant-CDH) Problem}

Theorem 1. The variant-CDH problem is hard if the co- $B D H$ problem is hard.

Proof. Suppose that there exists an oracle which can solve the variant-CDH problem with nonnegligible probability. We will prove that the oracle can help us to solve the co$\mathrm{BDH}$ problem with nonnegligible probability. Given any parameters of the co-BDH problem, $\left(P_{1}, a P_{1}, b P_{1}\right) \in \mathbb{G}_{1}^{3}$ and $\left(P_{2}, a P_{2}, c P_{2}\right) \in \mathbb{G}_{2}^{3}$, we input $\left(P_{1}, a P_{1}, b P_{1}\right)$ and $\left(P_{2}, a P_{2}\right)$ into the variant-CDH oracle. The oracle will output $a b P_{1}$. Then, we solve the co-BDH problem by computing $e\left(a b P_{1}, c P_{2}\right)=$ $e\left(P_{1}, P_{2}\right)^{a b c}$.

\subsection{The n-Linear Diffie-Hellman (n-LDH) Problem}

Theorem 2. The $n-L D H$ problem is hard if and only if the DCDH problem is hard.

Proof. (1) $n-\mathrm{LDH} \Rightarrow$ DCDH. Suppose that there exists an oracle which can solve the $n$-LDH problem with nonnegligible probability. We will prove that the oracle can help us to solve the DCDH problem with nonnegligible probability.
For any DCDH triple $(P, x P, y P)$, we convert them into the $n$-LDH oracle's input parameters which are shown in (2):

$$
\left[\begin{array}{ccccc}
x_{1} P & x_{2} P & \ldots & x_{n-1} P & x_{n} P \\
\perp & x_{2} x_{n+1} P & \ldots & x_{n-1} x_{n+1} P & x_{n} x_{n+1} P \\
x_{1} x_{n+2} P & \perp & \ldots & x_{n-1} x_{n+2} P & x_{n} x_{n+2} P \\
\vdots & \vdots & & \vdots & \vdots \\
x_{1} x_{2 n-1} P & x_{2} x_{2 n-1} P & \ldots & \perp & x_{n} x_{2 n-1} P \\
x_{1} x_{2 n} P & x_{2} x_{2 n} P & \ldots & x_{n-1} x_{2 n} P & \perp
\end{array}\right]
$$

We randomly pick $t_{2}, t_{3}, \ldots, t_{n} \in \mathbb{Z}_{q}^{*}$ and $Q_{1}, Q_{2}, \ldots$, $Q_{n-1} \in \mathbb{G}_{1}^{*}$, compute $Q_{n}=x P-Q_{1}-Q_{2}-\cdots-Q_{n-1}$, and set other parameters in (3):

$$
\left[\begin{array}{cccccc}
y P & t_{2} y P & t_{3} y P & \ldots & t_{n-1} y P & t_{n} y P \\
\perp & t_{2} Q_{1} & t_{3} Q_{1} & \ldots & t_{n-1} Q_{1} & t_{n} Q_{1} \\
Q_{2} & \perp & t_{3} Q_{2} & \ldots & t_{n-1} Q_{2} & t_{n} Q_{2} \\
\vdots & \vdots & \vdots & & \vdots & \vdots \\
Q_{n-1} & t_{2} Q_{n-1} & t_{3} Q_{n-1} & \ldots & \perp & t_{n} Q_{n-1} \\
Q_{n} & t_{2} Q_{n} & t_{3} Q_{n} & \ldots & t_{n-1} Q_{n} & \perp
\end{array}\right]
$$

Equation (2) is equal to (3); that is, $x_{1} P=y P, x_{2} P=$ $t_{2} x_{1} P=t_{2} y P, x_{3} P=t_{3} x_{1} P=t_{3} y P, \ldots, x_{n} P=t_{n} x_{1} P=$ $t_{n} y P$ in row $1, x_{2} x_{n+1} P=t_{2} x_{1} x_{n+1} P=t_{2} Q_{1}, x_{3} x_{n+1} P=$ $t_{3} x_{1} x_{n+1} P=t_{3} Q_{1}, \ldots, x_{n} x_{n+1} P=t_{n} x_{1} x_{n+1} P=t_{n} Q_{1}$ in row 2 (suppose that $x_{1} x_{n+1} P=Q_{1}$ ), $x_{1} x_{n+2} P=Q_{2}, x_{3} x_{n+2} P=$ $t_{3} x_{1} x_{n+2} P=t_{3} Q_{2}, \ldots, x_{n} x_{n+2} P=t_{n} x_{1} x_{n+2} P=t_{n} Q_{2}$ in row $3, \ldots$, and $x_{1} x_{2 n} P=Q_{n}, x_{2} x_{2 n} P=t_{2} x_{1} x_{2 n} P=t_{2} Q_{n}$, $x_{3} x_{2 n} P=t_{3} x_{1} x_{2 n} P=t_{3} Q_{n}, \ldots, x_{n-1} x_{2 n} P=t_{n-1} x_{1} x_{2 n} P=$ $t_{n-1} Q_{n}$ in row $n+1$. The oracle will output $\left(x_{n+1}+x_{n+2}+\right.$ $\left.x_{n+3}+\cdots+x_{2 n-1}+x_{2 n}\right) P$. Thus, we have that

$$
\begin{aligned}
x P & =Q_{1}+Q_{2}+\cdots+Q_{n-1}+Q_{n} \\
& =x_{1} x_{n+1} P+x_{1} x_{n+2} P+\cdots+x_{1} x_{2 n-1} P+x_{1} x_{2 n} P \\
& =\left(x_{n+1}+x_{n+2}+\cdots+x_{2 n-1}+x_{2 n}\right) x_{1} P \\
& =\left(x_{n+1}+x_{n+2}+\cdots+x_{2 n-1}+x_{2 n}\right) y P .
\end{aligned}
$$

From (4), we can get $x y^{-1} P=\left(x_{n+1}+x_{n+2}+\cdots+x_{2 n-1}+x_{2 n}\right) P$.

(2) $n$-LDH $\Leftarrow \mathrm{DCDH}$. Suppose that there exists an oracle which can solve the the DCDH problem with nonnegligible probability. We will prove that the oracle can help us to solve the $n$-LDH problem with nonnegligible probability, too.

For any $n-\mathrm{LDH}$ tuple in (2), we input $\left(x_{1} x_{n+2} P, x_{1} P\right)$, $\left(x_{1} x_{n+3} P, x_{1} P\right), \ldots,\left(x_{1} x_{2 n} P, x_{1} P\right)$, and $\left(x_{2} x_{n+1} P, x_{2} P\right)$ into the oracle. Then the oracle outputs $x_{n+2} P, x_{n+3} P, \ldots, x_{2 n} P$, and $x_{n+1} P$, respectively.

Finally, we can compute $x_{n+1} P+x_{n+2} P+\cdots+x_{2 n} P=$ $\left(x_{n+1}+x_{n+2}+\cdots+x_{2 n}\right) P$ to solve the $n$-LDH problem.

We use a way similar to [22] to prove the $n$-DLDH problem being hard. In the generic model, elements of $\mathbb{G}_{1}$, $\mathbb{G}_{2}$, and $\mathbb{G}_{T}$ are encoded as unique random strings, where $\mathbb{G}_{1}$ and $\mathbb{G}_{2}$ are additive groups and $\mathbb{G}_{T}$ is a multiplicative group. There is a bilinear pairing function $e: \mathbb{G}_{1} \times \mathbb{G}_{2} \rightarrow \mathbb{G}_{T}$. Let $S_{1}, S_{2}$, and $S_{T}$ be the sets of strings. The opaque encoding of the elements of $\mathbb{G}_{1}$ is modeled as an injective function 
$\zeta_{1}: \mathbb{Z}_{q} \rightarrow S_{1}$, where $S_{1} \subset\{0,1\}^{*}$, which maps all $a \in \mathbb{Z}_{q}$ to the string representation $\zeta_{1}(a)$ of $a P \in \mathbb{G}_{1}$. Analogous mapping $\zeta_{2}: \mathbb{Z}_{q} \rightarrow S_{2}$ and $\zeta_{T}: \mathbb{Z}_{q} \rightarrow S_{T}$ map all $a \in \mathbb{Z}_{q}$ to the string representation $\zeta_{2}(a)$ of $a P^{\prime} \in \mathbb{G}_{2}$ and $\zeta_{T}(a)$ of $g^{a} \in \mathbb{G}_{T}$, where $g=e\left(P, P^{\prime}\right)$.

\subsection{The Decisional n-Linear Diffie-Hellman (n-DLDH) Problem}

Theorem 3. Let $\mathscr{A}$ be an algorithm that solves the $n-D L D H$ problem in the generic bilinear group model with at most $q_{k}$ oracle queries. Let $x_{i}$ 's, $x_{n+j}$ 's, and $z \in \mathbb{Z}_{q}$ be chosen at random, where $1 \leq i, j \leq n$, and $n \geq 2, \zeta_{1}, \zeta_{2}$, and $\zeta_{T}$ are random encoding functions for $G_{1}, G_{2}$, and $G_{T}$, and $b \in\{0,1\}$ is a random bit. Let $w_{b}=z$ and $w_{1-b}=\sum_{j=1}^{n} x_{n+j} \bmod q$. The probability is

$$
\begin{aligned}
& \operatorname{Pr}\left[\begin{array}{c}
\mathscr{A}\left(\zeta_{1}, \zeta_{2}, \zeta_{T} ; 1, x_{i}{ }^{\prime} s, x_{i} x_{n+j}{ }^{\prime} s, w_{0}, w_{1}\right)=b: \\
\forall i, j, x_{i}, x_{n+j} \in \mathbb{Z}_{q}^{*}, \\
1 \leq i, j \leq n, i \neq j, n \geq 2, b \in\{0,1\}
\end{array}\right] \\
& =\frac{1}{2}+\frac{2\left(n^{2}+3+q_{k}\right)^{2}}{q} .
\end{aligned}
$$

Proof. $\mathscr{B}$ plays the following game with $\mathscr{A}$. $\mathscr{B}$ maintains the lists $L_{1}=\left\{\left(F_{1, s}, \zeta_{1, s}\right): s=0,1,2, \ldots, \tau_{1}-1\right\}$, $L_{2}=\left\{\left(F_{2, t}, \zeta_{2, t}\right): t=0,1,2, \ldots, \tau_{2}-1\right\}$, and $L_{T}=\left\{\left(F_{T, u}, \zeta_{T, u}\right): t=0,1,2, \ldots, \tau_{T}-1\right\}$. Let $X_{i}$ 's, $X_{i} X_{n+j}$ 's, $W_{0}$, and $W_{1}(\forall i, j, 1 \leq i, j \leq n, i \neq j)$ be indeterminate. All $F_{1, s}$ 's, $F_{2, t}$ 's, and $F_{T, u}$ 's $\in \mathbb{Z}_{q}\left[X_{i}\right.$ 's, $X_{i} X_{n+j}$ 's, $\left.W_{0}, W_{1}\right]$ are polynomials and $\zeta_{1, s}$ 's, $\zeta_{2, t}$ 's, $\zeta_{T, u}$ 's $\in\{0,1\}^{*}$ are distinct strings. At the beginning of the game, $\mathscr{B}$ sets $F_{1,0}=1, F_{1,1}=X_{1}$, $F_{1,2}=X_{2}, \ldots, F_{1, n}=X_{n}, F_{1, n^{2}+1}=W_{0}, F_{1, n^{2}+2}=W_{1}, F_{2,0}=1$, $F_{T, 0}=1$, and the following polynomials: $\left[\left(\perp, F_{1, n+1}=\right.\right.$ $\left.X_{2} X_{n+1}, \ldots, F_{1,2 n-2}=X_{n-1} X_{n+1}, F_{1,2 n-1}=X_{n} X_{n+1}\right),\left(F_{1,2 n}=\right.$ $\left.X_{1} X_{n+2}, \perp, \ldots, F_{1,3 n-3}=X_{n-1} X_{n+2}, F_{1,3 n-2}=X_{n} X_{n+2}\right), \ldots$, $\left(F_{1, n^{2}-2 n+3}=X_{1} X_{2 n-1}, F_{1, n^{2}-2 n+4}=X_{2} X_{2 n-1}, \ldots, \perp\right.$ , $\left.F_{1, n^{2}-n+1}=X_{n} X_{2 n-1}\right),\left(F_{1, n^{2}-n+2}=X_{1} X_{2 n}, F_{1, n^{2}-n+3}=\right.$ $\left.\left.X_{2} X_{2 n}, \ldots, F_{1, n^{2}}=X_{n-1} X_{2 n}, \perp\right)\right]$, where the symbol “ $\perp$ " means emptiness and $\mathscr{B}$ gives $\mathscr{A}$ the distinct strings $\zeta_{1,0}, \zeta_{1,1}, \ldots, \zeta_{1, n^{2}+2}, \zeta_{2,0}$, and $\zeta_{T, 0}$. In the initial list index, the numbers of the records in $L_{1}, L_{2}$, and $L_{T}$ are $\tau_{1}=n^{2}+3$, $\tau_{2}=1$, and $\tau_{T}=1$, respectively, where $\tau=\tau_{1}+\tau_{2}+\tau_{T}=n^{2}+5$. At any step in the game, $\mathscr{A}$ can make the group and pairing queries. $\mathscr{B}$ performs and responds to $\mathscr{A}$ as follows.

Group Action. $\mathscr{A}$ gives $\mathscr{B}$ two operands $\zeta_{1, s}, \zeta_{1, t}$ and a sign bit, where $0 \leq s, t<\tau_{1}$. $\mathscr{B}$ sets $F_{1, \tau_{1}} \leftarrow F_{1, s} \pm F_{1, t}$. If $F_{1, \tau_{1}}=F_{1, l}$ for some $l<\tau_{1}, \mathscr{B}$ sets $\zeta_{1, \tau_{1}} \leftarrow \zeta_{1, l}$. Otherwise, $\mathscr{B}$ sets $\zeta_{1, \tau_{1}}$ to be a string in $\{0,1\}^{*}$ distinct from $\zeta_{1,0}, \zeta_{1,1}, \ldots, \zeta_{1, \tau_{1}-1}$. Finally, $\mathscr{B}$ adds $\left(F_{1, \tau_{1}}, \zeta_{1, \tau}\right)$ to the list $L_{1}$, gives $\zeta_{1, \tau_{1}}$ to $\mathscr{A}$, and sets $\tau_{1} \leftarrow \tau_{1}+1$. The group action queries in $G_{2}$ and $G_{T}$ are simulated similarly.

Pairing. $\mathscr{A}$ gives $\mathscr{B}$ two operands $\zeta_{1, s}$ and $\zeta_{1, t}$ with $0 \leq s<\tau_{1}$ and $0 \leq t<\tau_{2}$. $\mathscr{B}$ sets the product $F_{T, \tau_{T}} \leftarrow F_{1, s} F_{2, t}$. If $F_{T, \tau_{T}}=$ $F_{T, l}$ for some $l<\tau_{T}$, $\mathscr{B}$ sets $\zeta_{T, \tau_{T}} \leftarrow \zeta_{T, l}$. Otherwise, $\mathscr{B}$ sets
$\zeta_{T, \tau_{T}}$ to be a string in $\{0,1\}^{*}$ distinct from $\zeta_{T, 0}, \zeta_{T, 1}, \ldots, \zeta_{T, \tau_{T}-1}$. Finally, $\mathscr{B}$ adds $\left(F_{T, \tau_{T}}, \zeta_{T, \tau_{T}}\right)$ to the list $L_{T}$, gives $\zeta_{T, \tau_{T}}$ to $\mathscr{A}$, and sets $\tau_{T} \leftarrow \tau_{T}+1$.

Consider the operation that $\mathscr{B}$ performs: (1) $\mathscr{B}$ adds/subtracts all polynomials in the list $L_{1}, L_{2}$, and $L_{T}$ by any $\mathscr{A}$ 's query. (2) $\mathscr{B}$ produces any of two polynomials in $L_{1}$ and $L_{2}$ to generate a new polynomial in $L_{T}$. For any variant $X_{n+j}$, it occurs within the monomials $X_{1} X_{n+j}, X_{2} X_{n+j}, \ldots, X_{n+j-1} X_{n+j}, X_{n+j+1} X_{n+j}, \ldots, X_{2 n} X_{n+j}$ in $L_{1}$ and $L_{T}$ lists and it occurs no monomial in $L_{2}$ list. Therefore, $\mathscr{B}$ cannot produce any polynomial that contains the monomial $c X_{n+j}$ 's in $G_{1}$ or $G_{2}$ and the monomial $F_{2, t} X_{n+j}$ 's in $G_{T}$ for any coefficient $c \neq 0$ and any nonzero monomial $F_{2, t}$ in $L_{2}$ in the available operations.

After at most $q$ queries, $\mathscr{A}$ terminates and returns a guess $\widehat{b} \in\{0,1\}$. The distinct values of operands provide no information to $\mathscr{A}$ because they are random bit strings. Therefore, the probability that $\mathscr{A}$ wins the game in the generic model is $1 / 2$.

However, when $\mathscr{B}$ randomly chooses $x_{i}$ 's, $x_{n+j}$ 's, and $z \in \mathbb{Z}$, sets $w_{b}=\sum_{j=1}^{n} x_{n+j}$ and $w_{1-b}=z$, and assigns $X_{1} \leftarrow x_{1}, X_{2} \leftarrow x_{2}, \ldots, X_{2 n} \leftarrow x_{2 n}, W_{b} \leftarrow w_{b}$, and $W_{1-b} \leftarrow w_{1-b}$, a nontrivial equality relation may occur and give $\mathscr{A}$ some information that is not revealed in the generic model; that is, for some $F_{1, s} \neq F_{1, t}$ (and $F_{T, s} \neq F_{T, t}$, resp.) and $s, t<\tau_{1}$ (and $s, t<\tau_{T}$, resp.), $F_{1, s}\left(x_{i}\right.$ 's, $x_{i} x_{n+j}$ 's, $\left.w_{0}, w_{1}\right)$ $=F_{1, t}\left(x_{i}{ }^{\prime} \mathrm{s}, x_{i} x_{n+j}\right.$ 's, $\left.w_{0}, w_{1}\right)\left(\right.$ and $F_{T, s}\left(x_{i}\right.$ 's, $x_{i} x_{n+j}$ 's, $\left.w_{0}, w_{1}\right)=$ $F_{T, t}\left(x_{i}\right.$ 's, $x_{i} x_{n+j}$ 's, $\left.w_{0}, w_{1}\right)$, resp.).

The probability of the occurrence is computed according to the following lemma.

Lemma 4 (see [32]). Let $q$ be prime and let $t \geq 1$. Let $F\left(X_{1}, X_{2}, \ldots, X_{k}\right) \in Z / q^{t}\left[X_{1}, X_{2}, \ldots, X_{k}\right]$ be a nonzero polynomial of total degree $d$. Then for random $x_{1}, x_{2}, \ldots, x_{k} \in$ $Z / q^{t}$, the probability that $F\left(x_{1}, x_{2}, \ldots, x_{k}\right)=0$ is at most $d / q$.

By Lemma 4, all polynomials in the $L_{1}$ have degree at most 2 , so that, for some $s, t$ and $F_{1, s} \neq F_{1, t}$, the probability of $F\left(x_{i}\right.$ 's, $x_{i} x_{n+j}$ 's, $\left.w_{0}, w_{1}\right)=F_{1, s}\left(x_{i}\right.$ 's, $x_{i} x_{n+j}$ 's, $\left.w_{0}, w_{1}\right)-F_{1, t}\left(x_{i}\right.$ 's, $x_{i} x_{n+j}$ 's, $\left.w_{0}, w_{1}\right)=0$ is at most $2 / q$. The degree of polynomials in the $L_{2}$ is 0 . All polynomials in the $L_{T}$ have degree at most 2 , so that, for some $s, t$ and $F_{T, s} \neq F_{T, t}$, the probability of $F^{\prime}\left(x_{i}\right.$ 's, $x_{i} x_{n+j}$ 's, $\left.w_{0}, w_{1}\right)=F_{T, s}\left(x_{i}\right.$ 's,$x_{i} x_{n+j}$ 's, $\left.w_{0}, w_{1}\right)-F_{T, t}\left(x_{i}\right.$ 's, $x_{i} x_{n+j}$ 's, $\left.w_{0}, w_{1}\right)=0$ is at most $2 / q$. Therefore, $\mathscr{A}$ wins the game with the probability $\varepsilon \leq 1 / 2+\left(\begin{array}{c}\tau_{1} \\ 2\end{array}\right)(2 / q)+\left(\begin{array}{c}\tau_{T} \\ 2\end{array}\right)(2 / q)$. Since $\tau_{1}+\tau_{T} \leq n^{2}+4+q_{k}$, we have $\varepsilon \leq 1 / 2+\left(n^{2}+4+q_{k}\right)^{2} / q$, where the advantage is not greater than $\mathcal{O}\left(q_{k}^{2} / q\right)$.

\section{Our Key Agreement Schemes}

In this section, we propose two conference key agreement schemes. The first scheme is designed for the situation where the users who register with a single TA (single domain) want to negotiate a session conference key. Furthermore, the second scheme makes it possible for the users in distinct groups who register with different TAs (interdomain) to negotiate a session conference key. In addition, we will prove 
the security of the two proposed schemes in Section 6 and compare them with others in Section 7.

\subsection{The Proposed Scheme in Single TA}

Setup. TA inputs a security parameter $\kappa$ into a setup algorithm which returns groups $\mathbb{G}_{1}, \mathbb{G}_{2}$, and $\mathbb{G}_{T}\left(\mathbb{G}_{1} \neq \mathbb{G}_{2}\right)$ of prime order $q$ with $q \in\{0,1\}^{\kappa}$, a suitable bilinear mapping $e$ : $\mathbb{G}_{1} \times \mathbb{G}_{2} \rightarrow \mathbb{G}_{T}$, generators $P \in \mathbb{G}_{1}, P^{\prime} \in \mathbb{G}_{2}$, and three hash functions $H:\{0,1\}^{*} \rightarrow \mathbb{G}_{1}, H_{1}: \mathbb{G}_{1} \rightarrow\{0,1\}^{l}, H_{2}:$ $\mathbb{G}_{1} \times\{0,1\}^{*} \rightarrow\{0,1\}^{l}$, and $H_{3}: \mathbb{G}_{1} \rightarrow\{0,1\}^{l}$ where $l$ is the output length of the hash functions. TA randomly generates a long-term private key $s \in \mathbb{Z}_{q}^{*}$ and the public key $\left(s P, s P^{\prime}\right)$ and then publishes $\left\langle q, e, \mathbb{G}_{1}, \mathbb{G}_{2}, \mathbb{G}_{T}, P, s P, P^{\prime}, s P^{\prime}, H, H_{1}, H_{2}, H_{3}\right\rangle$.

Extract. When a user $U_{i}$ registers a public identity (ID), such as an email address, with TA, TA will check whether the ID belongs to the user. If true, TA issues a long-term private key $s Q_{i}$ to $U_{i}$ where $Q_{i}=H$ (TA's ID $\| U_{i}$ 's ID) is $U_{i}$ 's public key.

Conference Key Agreement. Suppose that there are $n$ legal users $U_{1}, U_{2}, \ldots$, and $U_{n}$ who want to negotiate a conference key. Our conference key agreement scheme contains three rounds described as follows.

Round 1: every user $U_{i}(1 \leq i \leq n)$ randomly picks an integer $r_{i} \in \mathbb{Z}_{q}^{*}$ as a blinding factor, and then $U_{i}$ computes $b_{i}=r_{i}^{-1} P$ and broadcasts $b_{i}$ to all users who join this session. The flow is shown in Algorithm 1.

Round 2: after $U_{i}$ receives all $b_{j}$ 's $(1 \leq j \leq n, j \neq i)$, she/he randomly picks an integer $k_{i} \in \mathbb{Z}_{q}^{*}$ as an ephemeral key and computes $B_{i}=\left(k_{i} b_{1}, k_{i} b_{2}, \ldots, k_{i} b_{i-1}, \perp, k_{i} b_{i+1}, \ldots, k_{i} b_{n}\right)$, and then $U_{i}$ broadcasts $B_{i}$. The flow is shown in Algorithm 2 .

Round 3: for all $B_{i}$ 's in Round 2, we can rearrange them as shown in (7). When receiving $B_{j}$ 's, $U_{i}$ only stores $k_{j} b_{i}(1 \leq$ $j \leq n, j \neq i)$ and drops other useless information $k_{j} b_{t}$ 's $(1 \leq$ $j \leq n, 1 \leq t \leq n, t \neq i$ ). For example, $U_{1}$ stores column 1 and $U_{2}$ stores column 2 in (7). Then $U_{i}$ computes $K$ as follows:

$$
\begin{aligned}
K & =r_{i} \sum_{j=1}^{n} k_{j} b_{i} \\
& =r_{i} \cdot\left(k_{1} r_{i}^{-1}+k_{2} r_{i}^{-1}+\cdots+k_{i} r_{i}^{-1}+\cdots+k_{n} r_{i}^{-1}\right) P \\
& =\left(k_{1}+k_{2}+\cdots+k_{i}+\cdots+k_{n}\right) P .
\end{aligned}
$$

All $B_{i}$ 's in Round 2

\begin{tabular}{c|cccccc} 
& $U_{1}$ & $U_{2}$ & $\ldots$ & $U_{i}$ & $\ldots$ & $U_{n}$ \\
\hline$B_{1}$ & $\perp$ & $k_{1} r_{2}^{-1} P$ & $\ldots$ & $k_{1} r_{i}^{-1} P$ & $\ldots$ & $k_{1} r_{n}^{-1} P$ \\
$B_{2}$ & $k_{2} r_{1}^{-1} P$ & $\perp$ & $\ldots$ & $k_{2} r_{i}^{-1} P$ & $\ldots$ & $k_{2} r_{n}^{-1} P$ \\
$\vdots$ & $\vdots$ & $\vdots$ & $\ddots$ & $\vdots$ & $\vdots$ & $\vdots$ \\
$B_{i}$ & $k_{i} r_{1}^{-1} P$ & $k_{i} r_{2}^{-1} P$ & $\ldots$ & $\perp$ & $\ldots$ & $k_{i} r_{n}^{-1} P$ \\
$\vdots$ & $\vdots$ & $\vdots$ & $\vdots$ & $\vdots$ & $\ddots$ & $\vdots$ \\
$B_{n}$ & $k_{n} r_{1}^{-1} P$ & $k_{n} r_{2}^{-1} P$ & $\ldots$ & $k_{n} r_{i}^{-1} P$ & $\ldots$ & $\perp$
\end{tabular}

$U_{i}$ computes $\alpha=H_{1}(K), T_{i}=\alpha s Q_{i}+r_{i}^{-1} s P$, and $\beta_{i}=H_{2}\left(T_{i}, \alpha\right)$, and then she/he broadcasts $\left(T_{i}, \beta_{i}\right)$. When $U_{i}$ receives all $\left(T_{j}, \beta_{j}\right)$ 's $(1 \leq j \leq n, j \neq i)$, she/he first verifies all $\beta_{j}$ 's by checking if $H_{2}\left(T_{j}, \alpha\right)=\beta_{j}$ for each $j$. If they are true, $U_{i}$ randomly chooses $a_{1}, a_{2}, \ldots, a_{n} \in \mathbb{Z}_{q}^{*}$, computes $V_{i}=e\left(\sum_{i=1}^{n} a_{i} b_{i}+\alpha \sum_{i=1}^{n} a_{i} Q_{i}, s P^{\prime}\right)$, and verifies whether $V_{i}=$ $e\left(\sum_{i=1}^{n} a_{i} T_{i}, P^{\prime}\right)$ or not. If true, $U_{i}$ accepts $K$ and computes the session conference key SCK $=H_{3}(K)$. Algorithm 3 illustrates the flow in Round 3.

5.2. The Proposed Scheme in Distinct TAs. Our single domain conference key agreement scheme can be extended to an interdomain conference key agreement scheme. Interdomain means that there are distinct domains with different TAs', respectively. In this subsection, we present our interdomain conference key scheme. Assume that there are $m$ Trusted Authorities $\mathrm{TA}_{1}, \mathrm{TA}_{2}, \ldots$, and $\mathrm{TA}_{m}$ and $m$ user groups $G_{1}, G_{2}, \ldots$, and $G_{m}$ who register with the $m$ distinct TAs, respectively. In the proposed scheme, the users in $m$ different groups can negotiate a session conference key SCK via the following process.

Setup. $\mathrm{TA}_{i}, i \in\{1,2, \ldots, m\}$, inputs a security parameter $\kappa$ into a setup algorithm which returns two groups $\mathbb{G}_{1}, \mathbb{G}_{2}$, and $\mathbb{G}_{T}\left(\mathbb{G}_{1} \neq \mathbb{G}_{2}\right)$ of prime order $q \in\{0,1\}^{\kappa}$, a suitable bilinear mapping $e: \mathbb{G}_{1} \times \mathbb{G}_{2} \rightarrow \mathbb{G}_{T}$, generators $P \in \mathbb{G}_{1}$, $P^{\prime} \in \mathbb{G}_{2}$, and three hash functions $H:\{0,1\}^{*} \rightarrow \mathbb{G}_{1}$, $H_{1}: \mathbb{G}_{1} \rightarrow\{0,1\}^{l}, H_{2}: \mathbb{G}_{1} \times\{0,1\}^{*} \rightarrow\{0,1\}^{l}$, and $H_{3}: \mathbb{G}_{1} \rightarrow\{0,1\}^{l}$ where $l$ is the output length of the hash functions. $\mathrm{TA}_{i}$ randomly generates a long-term key $s_{i} \in \mathbb{Z}_{q}^{*}$ and public key $\left(s_{i} P, s_{i} P^{\prime}\right)$ and then publishes $\left\langle q, e, \mathbb{G}_{1}, \mathbb{G}_{2}, \mathbb{G}_{T}\right.$, $\left.P, s_{i} P, P^{\prime}, s_{i} P^{\prime}, H, H_{1}, H_{2}, H_{3}\right\rangle$.

Extract. When a user $U_{j}$ in group $i$ registers a public ID with $\mathrm{TA}_{i}, \mathrm{TA}_{i}$ will check whether the ID belongs to the user. If true, $\mathrm{TA}_{i}$ issues private key $s_{i} Q_{i, j}$ to the user, where $Q_{i, j}=H\left(\mathrm{TA}_{i}\right.$ 's ID $\| U_{i, j}$ 's ID) is the public key and $U_{i, j}$ denotes user $U_{j}$ who has registered with $\mathrm{TA}_{i}$.

Interdomain Conference Key Agreement. Suppose that users in $m$ distinct domains or groups want to negotiate a conference key. Let $n_{h}(1 \leq h \leq m)$ be the number of users in the $h$ th domain and $N$ be the number of the total users $\left(N=n_{1}+\right.$ $\left.n_{2}+\cdots+n_{m}\right)$. Our interdomain conference key agreement protocol contains three rounds where Round 1 and Round 2 are similar to those of the proposed single domain conference key protocol.

Round 1: every user $U_{i, j}$ randomly picks an integer $r_{i, j} \epsilon$ $\mathbb{Z}_{q}^{*}$ and then broadcasts $b_{i, j}=\left(r_{i, j}\right)^{-1} P$ to the users who join this session.

Round 2: after receiving $b_{h, l}$ 's $(1 \leq h \leq m, 1 \leq l \leq$ $\left.n_{h}, h \neq i, l \neq j\right), U_{i, j}$ computes $k_{i, j} b_{h, l}$, where $k_{i, j} \in_{R} \mathbb{Z}_{q}^{*}$ is $U_{i, j}$ 's ephemeral key, and broadcasts $B_{i, j}=\left(k_{i, j} b_{1,1}, k_{i, j} b_{1,2}, \ldots, k_{i, j} b_{1, n_{1}}, \ldots, k_{i, j} b_{i, 1}, \ldots, k_{i, j} b_{i, j-1}, \perp\right.$ , $\left.k_{i, j} b_{i, j+1}, \ldots, k_{i, j} b_{i, n_{i}}, \ldots, k_{i, j} b_{m, 1}, \ldots, k_{i, j} b_{m, n_{m}}\right)$.

Round 3: when receiving $B_{h, l}$ 's, $U_{i, j}$ only stores $k_{h, l} b_{i, j}$ 's $\left(1 \leq h \leq m, 1 \leq l \leq n_{h}, h \neq i\right.$, and $\left.l \neq j\right)$ and drops other useless information $k_{h, l} b_{u, v}$ 's $\left(1 \leq h \leq m, 1 \leq l \leq n_{h}, 1 \leq u \leq\right.$ 


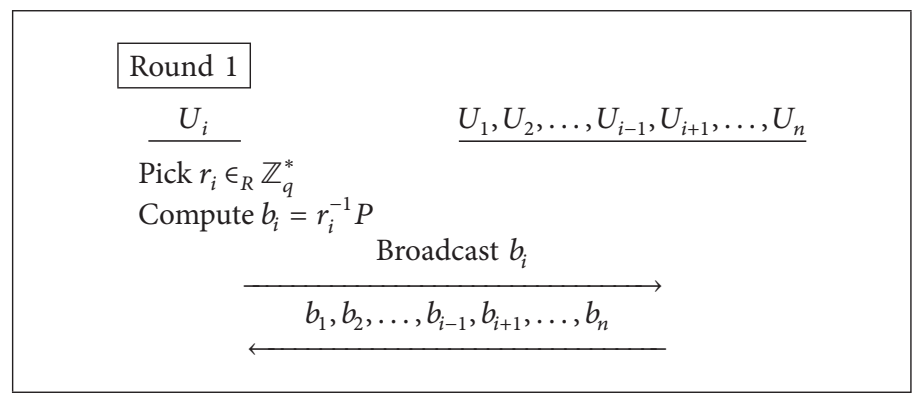

Algorithm 1: Round 1 of the first conference key protocol.

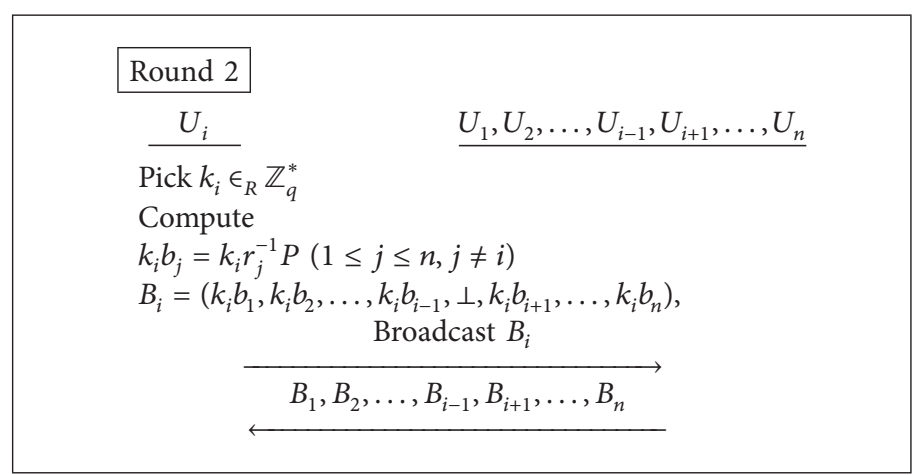

Algorithm 2: Round 2 of the first conference key protocol.

$m, 1 \leq v \leq n_{u}, u \neq i$, and $v \neq j$ ). Then $U_{i, j}$ computes every domain's key $K_{h}$ as follows:

$$
\begin{aligned}
K_{h} & =r_{i, j} \sum_{t=1}^{n_{h}} k_{h, t} b_{i, j} \\
& =r_{i, j}\left(k_{h, 1} r_{i, j}^{-1}+k_{h, 2} r_{i, j}^{-1}+\cdots+k_{h, n_{h}} r_{i, j}^{-1}\right) P \\
& =\left(k_{h, 1}+k_{h, 2}+\cdots+k_{h, n_{h}}\right) P .
\end{aligned}
$$

$U_{i, j}$ computes $K=\sum_{h=1}^{m} K_{h}, \alpha=H_{1}(K), T_{i, j}=\alpha s_{i} Q_{i, j}+$ $r_{i, j}^{-1} s_{i} P$, and $\beta_{i, j}=H_{2}\left(T_{i, j}, \alpha\right)$, and then she/he broadcasts $\left(T_{i, j}, \beta_{i, j}\right)$. When $U_{i, j}$ receives all messages, $U_{i, j}$ first verifies all $\beta_{h, l}$, s by examining if $H_{2}\left(T_{h, l}, \alpha\right)=\beta_{h, l}$ for each $h$ and $l(1 \leq$ $h \leq m, 1 \leq l \leq n_{h}, h \neq i$, and $\left.l \neq j\right)$. If all $\beta_{h, l}$ 's are correct, $U_{i, j}$ randomly chooses $N$ integers $a_{h, l}$ 's, where each $a_{h, l} \in \mathbb{Z}_{q}^{*}$, computes $V_{h, l}=\prod_{h=1}^{m} e\left(\sum_{l=1}^{n_{h}} a_{h, l} b_{h, l}+\alpha \sum_{l=1}^{n_{h}} a_{h, l} Q_{h, l}, s_{h} P^{\prime}\right)$, and checks if $V_{h, l}=e\left(\sum_{h=1}^{m} \sum_{l=1}^{n_{h}} a_{h, l} T_{h, l}, P^{\prime}\right)$. If it is true, $U_{i, j}$ accepts $K$ and computes the session conference key SCK $=$ $H_{3}(K)$.

\section{Security Proof}

Bellare-Rogaway random oracle model [12, 33], which was extended by Blake-Wilson et al. [34], is suitably modified and adapted in analyzing the security of key agreement protocols like those in the literatures $[9,13]$. In this section, we modify Bellare-Rogaway random oracle model and adopt the similar concepts and definitions in [8] to set our security game.
Definition 5 (game environment). Let adversary $\mathscr{A}$ be a probabilistic polynomial time Turing machine and $\mathscr{B}$ a simulator to simulate this game for $\mathscr{A}$. Let $\mathscr{I}=\left\{U_{1}, U_{2}, \ldots, U_{q}\right\}$ be all users and $\mathcal{U}$ the group users who follow our first identitybased conference key $(\mathscr{I} \mathscr{D} \mathscr{C} \mathscr{K})$ scheme, where $q$ is the order of $\mathbb{G}_{1}$ and $\mathscr{U} \subseteq\left\{U_{1}, U_{2}, \ldots, U_{q}\right\}$. In the game, we allow $\mathscr{A}$ to make the following types of queries.

(1) Execute $\left(\Pi_{\mathscr{U}}^{s}\right)$ : when $\mathscr{A}$ makes the Execute query, $\mathscr{B}$ simulates $\mathcal{U}$ to run the first $\mathscr{I} \mathscr{D} \mathscr{C} \mathscr{K}$ protocol $\Pi$ (Section 5.1) and responds with all public messages (i.e., $\left(b_{i}, B_{i}, T_{i}, \beta_{i}\right)$ 's for all $U_{i}$ 's $\left.\in \mathcal{U}\right)$ in the $s$ th session.

(2) $\operatorname{Send}\left(\Pi_{\widetilde{\mathscr{U}}}^{s}, m\right)$ : when $\mathscr{A}$ makes the Send query with a set of users $\widetilde{\mathcal{U}} \subset \mathcal{U}$ and a message $m$ which is the set of $\left(b_{j}, B_{j}, T_{j}, \beta_{j}\right)$ 's broadcast by the users in $\widetilde{\mathcal{U}}, \mathscr{B}$ simulates all $U_{i}$ 's $\in \mathscr{U}-\widetilde{\mathscr{U}}$ to interact with $\mathscr{A}$ by broadcasting the messages $\left(b_{i}, B_{i}, T_{i}, \beta_{i}\right)$ 's of $U_{i}$ 's in the sth session.

(3) Reveal $\left(\Pi_{\mathcal{U}}^{s}\right): \mathscr{B}$ reveals the session conference key SCK which was held by $\mathcal{U}$ in the sth session.

(4) $\operatorname{Corrupt}\left(U_{i}\right): \mathscr{B}$ responds with the long-term private key of $U_{i}$.

(5) $\operatorname{Test}\left(\Pi_{\mathcal{U}}^{s}\right)$ : when $\mathscr{A}$ makes the Test query, $\mathscr{B}$ returns the broadcast messages of the sth session and gives the adversary either the session key of the sth session or a random string. $\mathscr{A}$ then outputs a bit to decide whether the string is the session key or not. 


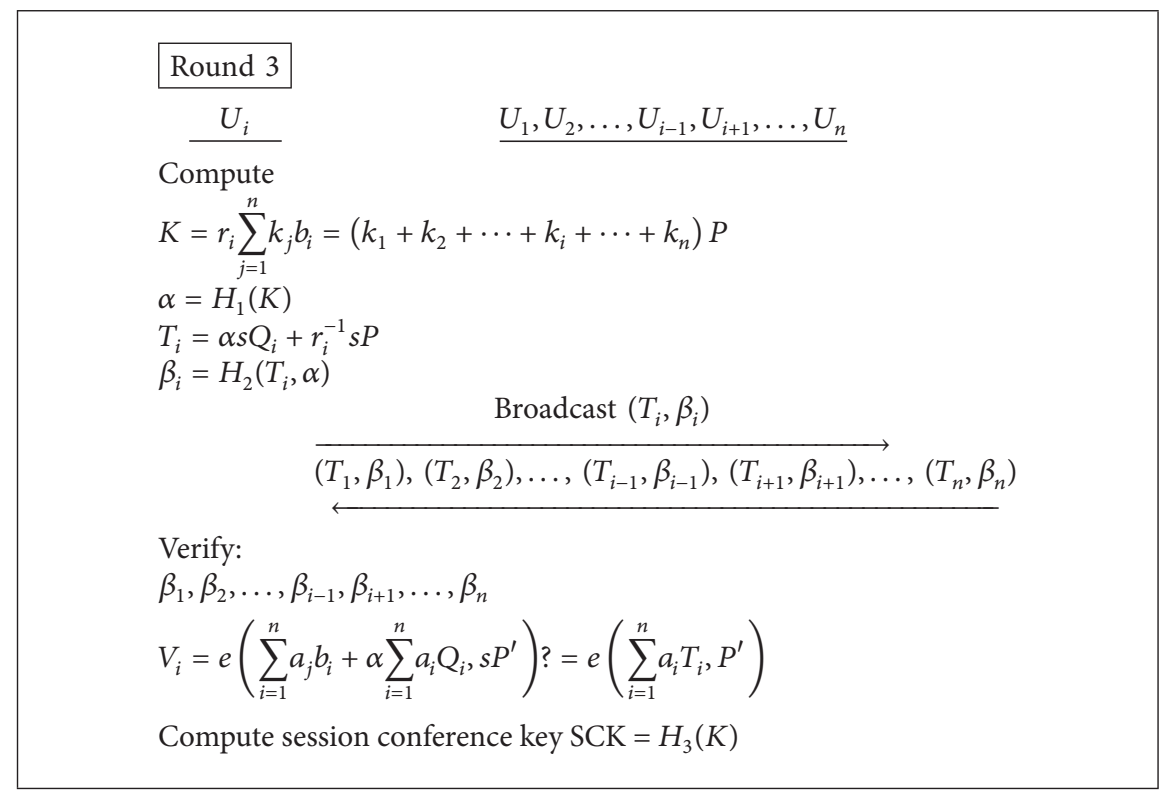

Algorithm 3: Round 3 of the first conference key protocol.

(6) $H(\cdot)$ : when a participant inputs a string to $H$, it responds with the hashed value of the string and the hashed value will be recorded.

(7) $H_{1}(\cdot)$ : when a participant inputs a message $m \in \mathbb{G}_{1}$ to $H_{1}$, it responds with the hashed value of $m$ and the hashed value will be recorded, too.

(8) $H_{2}(\cdot)$ : when a participant inputs $\left(m_{1}, m_{2}\right)$, where $m_{1} \in \mathbb{G}_{1}$ and $m_{2} \in\{0,1\}^{*}$, to $H_{2}$, it responds with the hashed value of $\left(m_{1}, m_{2}\right)$ and the hashed value will be recorded, too.

\subsection{Correctness}

Theorem 6 (correctness). In the presence of a benign adversary $\mathscr{A}$, all the parties always accept holding the same session conference key, which is distributed randomly and uniformly in $\{0,1\}^{\kappa}$, where $\kappa$ is the security parameter.

Proof. Every user $U_{i}$ can generate a valid message $\left(b_{i}, B_{i}, T_{i}, \beta_{i}\right)$ by following our proposed single domain scheme (Section 5.1), verify the correctness of the message $V_{i}=e\left(\sum_{i=1}^{n} a_{i} b_{i}+\alpha \sum_{i=1}^{n} a_{i} Q_{i}, s P^{\prime}\right)=e\left(\left(a_{1} r_{1}^{-1}+a_{2} r_{2}^{-1}+\right.\right.$ $\left.\left.\cdots+a_{n} r_{n}^{-1}\right) P+\left(\alpha a_{1} Q_{1}+\alpha a_{2} Q_{2}+\cdots+\alpha a_{n} Q_{n}\right), s P^{\prime}\right)$ $=e\left(a_{1}\left(\alpha s Q_{1}+r_{1}^{-1} s P\right)+a_{2}\left(\alpha s Q_{2}+r_{2}^{-1} s P\right)+\cdots+\right.$ $\left.a_{n}\left(\alpha s Q_{n}+r_{n}^{-1} s P\right), P^{\prime}\right)=e\left(\sum_{i=1}^{n} a_{i}\left(\alpha s Q_{i}+r_{i}^{-1} s P\right), P^{\prime}\right)=$ $e\left(\sum_{i=1}^{n} a_{i} T_{i}, P^{\prime}\right)$, and negotiate a common session conference key SCK $=H_{3}(K)$.

In our proposed interdomain scheme (Section 5.2), $U_{i j}$ can generate a valid message $\left(b_{i j}, B_{i j}, T_{i j}, \beta_{i j}\right)$ and verify the correctness of the message because

$$
\begin{aligned}
& V_{h, l}=\prod_{h=1}^{m} e\left(\sum_{l=1}^{n_{h}} a_{h, l} b_{h, l}+\alpha \sum_{l=1}^{n_{h}} a_{h, l} Q_{h, l}, s_{h} P^{\prime}\right) \\
& =e\left(\left(a_{1,1} r_{1,1}^{-1}+a_{1,2} r_{1,2}^{-1}+\cdots+a_{1, n_{1}} r_{1, n_{1}}^{-1}\right) P\right. \\
& \left.+\left(\alpha a_{1,1} Q_{1,1}+\alpha a_{1,2} Q_{1,2}+\cdots+\alpha a_{1, n_{1}} Q_{1, n_{1}}\right), s_{1} P^{\prime}\right) \\
& \cdot e\left(\left(a_{2,1} r_{2,1}^{-1}+a_{2,2} r_{2,2}^{-1}+\cdots+a_{2, n_{2}} r_{2, n_{2}}^{-1}\right) P\right. \\
& +\left(\alpha a_{2,1} Q_{2,1}+\alpha a_{2,2} Q_{2,2}+\cdots+\alpha a_{2, n_{2}} Q_{2, n_{2}}\right), \\
& \left.s_{2} P^{\prime}\right) \\
& \cdot e\left(\left(a_{m, 1} r_{m, 1}^{-1}+a_{m, 2} r_{m, 2}^{-1}+\cdots+a_{m, n_{m}} r_{m, n_{m}}^{-1}\right) P\right. \\
& +\left(\alpha a_{m, 1} Q_{m, 1}+\alpha a_{m, 2} Q_{m, 2}+\cdots+\alpha a_{m, n_{m}} Q_{m, n_{m}}\right), \\
& \left.s_{m} P^{\prime}\right) \\
& =e\left(a_{1,1}\left(\alpha s_{1} Q_{1,1}+r_{1,1}^{-1} s_{1} P\right)\right. \\
& +a_{1,2}\left(\alpha s_{1} Q_{1,2}+r_{1,2}^{-1} s_{1} P\right)+\cdots \\
& \left.+a_{1, n_{1}}\left(\alpha s_{1} Q_{1, n_{1}}+r_{1, n_{1}}^{-1} s_{1} P\right), P^{\prime}\right) \\
& \cdot e\left(a_{2,1}\left(\alpha s_{2} Q_{2,1}+r_{2,1}^{-1} s_{2} P\right)+a_{2,2}\left(\alpha s_{2} Q_{2,2}+r_{2,2}^{-1} s_{2} P\right)\right. \\
& \left.+a_{2, n_{2}}\left(\alpha s_{2} Q_{2, n_{2}}+r_{2, n_{2}}^{-1} s_{2} P\right), P^{\prime}\right) \\
& \cdot e\left(a_{m, 1}\left(\alpha s_{m} Q_{m, 1}+r_{m, 1}^{-1} s_{m} P\right)\right. \\
& +a_{m, 2}\left(\alpha s_{m} Q_{m, 2}+r_{m, 2}^{-1} s_{m} P\right)
\end{aligned}
$$




$$
\begin{aligned}
& \left.\quad+\cdots+a_{m, n_{m}}\left(\alpha s_{m} Q_{m, n_{m}}+r_{m, n_{m}}^{-1} s_{m} P\right), P^{\prime}\right) \\
& =e\left(\sum_{h=1}^{m} \sum_{l=1}^{n_{h}} a_{h, l}\left(\alpha s_{h} Q_{h, l}+r_{h, l}^{-1} s_{h} P\right), P^{\prime}\right) \\
& =e\left(\sum_{h=1}^{m} \sum_{l=1}^{n_{h}} a_{h, l} T_{h, l}, P^{\prime}\right) .
\end{aligned}
$$

6.2. Known Session Key Security. After given broadcast messages and previous session keys according to the $\mathscr{I} \mathscr{D} \mathscr{C} \mathscr{K}$ scheme, an adversary makes a Test query and then receives a random string or a current session key. The adversary can continue asking for broadcast messages and other session keys. If no polynomial-time adversary can decide whether the received string is the current session key or not with nonnegligible advantage, we say that the $\mathscr{I} \mathscr{D} \mathscr{C} \mathscr{K}$ scheme satisfies known session key security.

Definition 7 (known session key security). An $\mathscr{I} \mathscr{D} \mathscr{C} \mathscr{K}$ scheme is with known session key security if no polynomialtime adversary can decide if a challenge string is a current session key or a random string under the knowledge of previous session keys with the probability at least $(\varepsilon+1 / 2)$ where $\varepsilon$, called the advantage, is nonnegligible.

Theorem 8. If an adversary $\mathscr{A}$ can $\left(t, q_{E}, q_{S}, q_{C}, q_{R}, q_{H_{1}} \varepsilon\right)$ decide whether the string received from a Test query is the session key SCK held by $\Pi_{U}^{\ell}$ or not with advantage at least $\varepsilon$, where $t$ is the running time and $q_{E}, q_{S}, q_{C}, q_{R}$, and $q_{H_{1}}$ are the numbers of making Execute queries, Send queries, Corrupt queries, Reveal queries, and $H_{1}$ queries, respectively, there exists an algorithm which can solve the $n-D L D H$ problem with advantage at least $\varepsilon^{\prime}$ in time $t^{\prime}$, where

$$
\begin{aligned}
\varepsilon^{\prime} \geq & \frac{\varepsilon}{q_{0}} \\
t^{\prime} \approx & t+q_{E} \mathcal{O}\left(t_{E}\right)++q_{S} \mathcal{O}\left(t_{S}\right)+q_{C} \mathcal{O}\left(t_{C}\right) \\
& +q_{R} \mathcal{O}\left(t_{R}\right)+q_{H_{1}} \mathcal{O}\left(t_{H_{1}}\right)+\mathcal{O}\left(n^{2}\right)
\end{aligned}
$$

$q_{0}=q_{E}+q_{S}+q_{C}+q_{R}+q_{H}, t_{E}, t_{S}, t_{C}, t_{R}$, and $t_{H_{1}}$ are the computing time of the Execute oracle, the Send oracle, the Corrupt oracle, the Reveal oracle, and $H_{1}$ oracle, respectively.

Proof. Initially, we construct a simulator $\mathscr{B}$ which prepares the pairing parameters and simulates the system as follows. $\mathscr{B}$ randomly picks $s \in \mathbb{Z}_{q}^{*}$ as the system master private key and computes $\left(s P, s P^{\prime}\right)$ as the system master public key. $\mathscr{B}$ computes each user's long-term public/private key pair $\left(Q_{i}, s Q_{i}\right) . \mathscr{B}$ allows $\mathscr{A}$ to make the following queries

(i) $\operatorname{Execute}\left(\Pi_{\mathcal{U}}^{t}\right): \mathscr{A}$ can request $\mathcal{U}$ that is a set of users who are chosen by itself to run the key agreement protocol in session $t$. $\mathscr{B}$ follows the protocol (Section 5.1) to produce every $\left(r_{i}, b_{i}, B_{i}, T_{i}, \beta_{i}, k_{i}\right)$, and
$K=\sum_{U_{i} \in \mathscr{U}} k_{i} P$, and then records them. Finally, $\mathscr{B}$ responds every $\left(b_{i}, B_{i}, T_{i}, \beta_{i}\right)$ to $\mathscr{A}$, where $U_{i} \in \mathcal{U}$.

(ii) $\operatorname{Send}\left(\Pi_{\widetilde{\mathscr{U}}}^{t}, m\right)$ : if $\mathscr{A}$ actively broadcasts the messages of users $\widetilde{\mathcal{U}} \subset \mathcal{U}$ to run the key agreement protocol in session $t, \mathscr{B}$ follows the protocol (Section 5.1) to produce every $\left(r_{i}, b_{i}, B_{i}, T_{i}, \beta_{i}, k_{i}\right)$ and generate the session conference key in the end of the protocol and then records them. Finally, $\mathscr{B}$ responds $\left(b_{i}, B_{i}, T_{i}, \beta_{i}\right)$ to $\mathscr{A}$ for each $U_{i} \in \mathscr{U}-\widetilde{\mathscr{U}}$.

(iii) Reveal $\left(\Pi_{\mathcal{U}}^{t}\right)$ : if $\Pi_{\mathscr{U}}^{t}$ does not exist, $\mathscr{B}$ creates $\Pi_{\mathscr{U}}^{t} \cdot \mathscr{B}$ returns the session conference key SCK $=\mathrm{H}_{3}(K)$.

(iv) $\operatorname{Corrupt}\left(U_{i}\right): \mathscr{B}$ returns $\left(Q_{i}, s Q_{i}\right)$ to $\mathscr{A}$.

(v) $H_{1}(m)$ : after given $m \in \mathbb{G}_{1}, \mathscr{B}$ randomly chooses $\alpha \epsilon$ $\{0,1\}^{\lambda}$, returns $H_{1}(m)=\alpha$, and stores $(m, \alpha)$ in a list, called $\mathrm{H}_{1}$-list.

(vi) $\operatorname{Test}\left(\Pi_{\mathcal{U}^{*}}^{\ell}\right): \mathscr{B}$ guesses that $\mathscr{A}$ will send a Test query at the $h$ th session in advance. If $\mathscr{A}$ makes a Test query at the $\ell$ th session, where $\ell \neq h, \mathscr{B}$ randomly answers "YES" or "NO" to the $n$-DLDH problem. When $\mathscr{A}$ makes a Test query at the $\ell$ th session, where $\ell=h, \mathscr{B}$ checks whether there exists $U_{i}$ which has been corrupted or not, where $U_{i} \in \mathscr{U}^{*}$ and $\left|\mathcal{U}^{*}\right|=$ $n$. If one of them has been corrupted, $\mathscr{B}$ returns $\perp$ and aborts the game. Otherwise, $\mathscr{B}$ is given the parameters of an instance of the $n$-DLDH problem, $Z, P$, and $\left(x_{i} P, x_{i} x_{n+j} P\right)$ 's for all $i, j$ with $1 \leq i, j \leq n$, and $i \neq j$, and takes the advantage of $\mathscr{A}$ to decide whether $Z=\left(x_{n+1}+x_{n+2}+\cdots+x_{2 n}\right) P$ or not. $\mathscr{B}$ sets the public messages $\left(b_{i}^{*}, B_{i}^{*}, T_{i}^{*}, \beta_{i}^{*}\right)$ of every user in $\mathcal{U}^{*}$ in the $\ell$ th session as follows.

First, $\mathscr{B}$ forms $b_{1}^{*}=x_{1} P, b_{2}^{*}=x_{2} P, \ldots, b_{n}^{*}=x_{n} P$, $K^{*}=Z, \alpha^{*}=H_{1}\left(K^{*}\right)$, and prepares $B_{i}^{*}=\left(x_{1} x_{n+i} P\right.$, $\left.x_{2} x_{n+i} P, \ldots, x_{i-1} x_{n+i} P, \perp, x_{i+1} x_{n+i} P, \ldots, x_{n} x_{n+i} P\right)$, for each $U_{i}^{*} \in \mathcal{U}^{*}$; that is,

$$
\begin{array}{c|cccccc} 
& U_{1}^{*} & U_{2}^{*} & \ldots & U_{i}^{*} & \ldots & U_{n}^{*} \\
\hline B_{1}^{*} & \perp & x_{2} x_{n+1} P & \ldots & x_{i} x_{n+1} P & \ldots & x_{n} x_{n+1} P \\
B_{2}^{*} & x_{1} x_{n+2} P & \perp & \ldots & x_{i} x_{n+2} P & \ldots & x_{n} x_{n+2} P \\
\vdots & \vdots & \vdots & \ddots & \vdots & \vdots & \vdots \\
B_{i}^{*} & x_{1} x_{n+i} P & x_{2} x_{n+i} P & \ldots & \perp & \ldots & x_{n} x_{n+i} P \\
\vdots & \vdots & \vdots & \vdots & \vdots & \ddots & \vdots \\
B_{n}^{*} & x_{1} x_{2 n} P & x_{2} x_{2 n} P & \ldots & x_{i} x_{2 n} P & \ldots & \perp
\end{array}
$$

$\mathscr{B}$ sets $T_{i}^{*}=s \alpha^{*} Q_{i}+s x_{i} P$ and computes $\beta_{i}^{*}=$ $H_{2}\left(T_{i}^{*}, \alpha^{*}\right)$ and $\mathrm{SCK}^{*}=H_{3}\left(K^{*}\right)$, where $1 \leq i \leq n$. $\mathscr{B}$ responds every $\left(b_{i}^{*}, B_{i}^{*}, T_{i}^{*}, \beta_{i}^{*}\right)$ and $\mathrm{SCK}^{*}$ to $\mathscr{A}$.

$\mathscr{A}$ can continue making the queries of Execute $\left(\Pi_{u}^{t}\right)$, $\operatorname{Send}\left(\Pi_{\mathcal{U}}^{t}\right)$, Reveal $\left(\Pi_{\mathcal{U}}^{t}\right)$, and $\operatorname{Corrupt}\left(U_{i}\right)$, where $t \neq \ell$ and $U_{i} \notin \mathcal{U}^{*}$, until $\mathscr{A}$ outputs a bit $b$. If SCK ${ }^{*}$ is the key in session $\ell$ from $\mathscr{A}$ 's point of view, $\mathscr{A}$ will output $b=1$; otherwise, $b=0$. If $b=1, \mathscr{B}$ outputs "YES"; that is, $Z=\left(x_{n+1}+x_{n+2}+\cdots+x_{2 n}\right) P$; otherwise, $\mathscr{B}$ outputs "NO".

If the adversary can compromise known session key security of the scheme with advantage at least $\varepsilon, \mathscr{B}$ can 
solve the $n$-DLDH problem with the advantage at least $\varepsilon^{\prime} \geq$ $\left(1 / q_{0}\right)(1 / 2+\varepsilon)+(1 / 2)\left(\left(q_{0}-1\right) / q_{0}\right)-1 / 2=\varepsilon / q_{0}$.

By Theorem 8 , we can solve the $n$-DLDH problem in polynomial time with nonnegligible advantage if there exists a polynomial-time adversary that can break the known session key security with nonnegligible advantage of the proposed single domain key agreement scheme.

As for the proof of the interdomain case, we can let $n$ be the number of the total users from all domains; that is, $n=N$. Then, by the proof of Theorem 8 , the $n$-DLDH problem can be solved if the adversary can distinguish the session key from a random string in the proposed interdomain key agreement scheme.

6.3. Key-Compromise Impersonation. An adversary is given all users' long-term keys by making Corrupt queries except the one that he claims to impersonate. If no adversary can output the correct messages of the user with nonnegligible probability, the $\mathscr{I} \mathscr{D} \mathscr{C} \mathscr{K}$ scheme can withstand keycompromise impersonation.

Definition 9 (key-compromise impersonation). An $\mathscr{I} \mathscr{D} \mathscr{C} \mathscr{K}$ scheme can withstand key-compromise impersonation if no adversary can have nonnegligible probability to impersonate a user without the long-term private key of the user.

Lemma 10 (the forking lemma [35]). Let $T_{i}=\alpha s Q_{i}+r_{i}^{-1} s P$ be a valid authentic parameter of user $U_{i}$, where $s Q_{i}$ is $U_{i}$ 's private key, $s P$ is TA's public key, $r_{i}^{-1}$ is randomly chosen by $U_{i}$, and $\alpha$ is a hashed value of $K$ shared by all users. Let $\mathscr{A}$ be $a$ probabilistic polynomial time Turing machine. Given only the public data of the key agreement scheme as input, if $\mathscr{A}$ can find, with nonnegligible probability, a valid authentic parameter $T_{i}$ with $\alpha$, then, with nonnegligible probability, a replay of this machine, with the same random tape and a different value returned by the random oracle, can output two valid authentic parameters $T_{i}$ with $\alpha$ and $T_{i}^{\prime}$ with $\alpha^{\prime}$, such that $\alpha \neq \alpha^{\prime}$.

Lemma 11 (the splitting lemma [36]). Let $A \subset X \times Y$ such that $\operatorname{Pr}[(x, y) \in A]=|A| /(|X| \times|Y|) \geq \delta$. For any $\rho<\delta$, define $B=\{x \in X \mid \operatorname{Pr}[(x, y) \in A] \geq \delta-\rho\}$ and $\bar{B}=X \backslash B$. Then the following statements hold:

(1) $\operatorname{Pr}[x \in B]=|B| /|X| \geq \rho$,

(2) $\forall x \in B, \operatorname{Pr}[(x, y) \in A] \geq \delta-\rho$,

(3) $\operatorname{Pr}[x \in B \mid(x, y) \in A] \geq \rho / \delta$.

Lemma 12. Assume that $e\left(\sum_{i=1}^{n} a_{i} b_{i}+\alpha \sum_{i=1}^{n} a_{i} Q_{i}, s P^{\prime}\right)=$ $e\left(\sum_{i=1}^{n} a_{i} T_{i}, P^{\prime}\right)$. Let $E$ be an event that occurs if there is at least one $T_{i}$ such that $e\left(a_{i} b_{i}+\alpha a_{i} Q_{i}, s P^{\prime}\right) \neq e\left(a_{i} T_{i}, P^{\prime}\right)$. Then, the probability $\operatorname{Pr}[E] \leq 1 / 2^{q}$, where $q$ is a security parameter.

Proof. The proof is using the technique of the small exponents test in [37]. If $e\left(a_{i} b_{i}+\alpha a_{i} Q_{i}, s P^{\prime}\right) \neq e\left(a_{i} T_{i}, P^{\prime}\right)$ for some $i$, then $T_{i} \neq s b_{i}+s \alpha Q_{i}$. That is, there exists $c_{i} \neq 0(\bmod q)$ such that $T_{i}=s b_{i}+s \alpha Q_{i}+c_{i} P$.

Let $T_{j}=s b_{j}+s \alpha Q_{j}+c_{j} P$ where $\forall j \in\{1, \ldots, n\}-\{i\}$ and $c_{j} \in\{0, \ldots, q-1\}$. As $e\left(\sum_{i=1}^{n} a_{i} b_{i}+\alpha \sum_{i=1}^{n} a_{i} Q_{i}, s P^{\prime}\right)=$ $e\left(\sum_{i=1}^{n} a_{i} T_{i}, P^{\prime}\right), \sum_{i=1}^{n} c_{i} a_{i} \equiv 0(\bmod q)$. Hence, $a_{i} \equiv-c_{i}^{-1}\left(c_{1} a_{1}+\right.$ $\left.c_{2} a_{2}+\cdots+c_{i-1} a_{i-1}+c_{i+1} a_{i+1}+\cdots+c_{n} a_{n}\right)(\bmod q)$. Since $a_{i}$ is randomly chosen from $\mathbb{Z}_{q}^{*}, \operatorname{Pr}[E] \leq 1 / 2^{q}$.

Theorem 13. If an adversary $\mathscr{A}$ can $\left(t, q_{S}, q_{C}, q_{R}, q_{H}, q_{H_{1}}, \varepsilon\right)$ impersonate a user $U_{i}^{*}$ without the long-term private key of $U_{i}^{*}$ with probability at least $\varepsilon$, where $t$ is the running time, $q_{S}$, $q_{C}, q_{R}, q_{H}$, and $q_{H_{1}}$ are the numbers of making Send queries, Corrupt queries, Reveal queries, $H$ queries, and $H_{1}$ queries, respectively, there exists an algorithm to solve the variant- $\mathrm{CDH}$ problem with probability at least $\varepsilon^{\prime}$ in time $t^{\prime}$, where

$$
\begin{gathered}
\varepsilon^{\prime} \geq\left(\frac{(1 /|\mathcal{U}|)(1-1 /|\mathcal{U}|)\left(1-1 / 2^{h}\right)\left(\varepsilon-1 / 2^{q}\right)}{2}\right)^{2}, \\
t^{\prime} \approx 2\left(t+q_{S} \mathcal{O}\left(t_{S}\right)+q_{C} \mathcal{O}\left(t_{C}\right)+q_{R} \mathcal{O}\left(t_{R}\right)\right. \\
\left.+q_{H} \mathcal{O}\left(t_{H}\right)+q_{H_{1}} \mathcal{O}\left(t_{H_{1}}\right)\right)+\mathcal{O}\left(n^{2}\right),
\end{gathered}
$$

$h$ is the length of $H_{1}$ 's output, $\mathcal{U}$ is a set of users, and $t_{H}$ is the computing time of $H$ oracle.

Proof. At first, $\mathscr{B}$ inputs $\kappa$ to generate pairing parameters and a variant-CDH tuple $\left(P, a P, b P, P^{\prime}, a P^{\prime}\right) \in \mathbb{G}_{1}$, where $a, b \in_{R} \mathbb{Z}_{q}^{*}$. We will show that $\mathscr{B}$ can solve the variant-CDH problem with the assistance of an adversary $\mathscr{A}$. $\mathscr{B}$ 's task is to compute and output the value $c P=a b P$.

$\mathscr{B}$ simulates the system as follows. We define $\left(a P, a P^{\prime}\right)$ as the system master public key and $\mathscr{B}$ does not know the master private key $a$. $H$ and $H_{1}$ are two random oracles simulated by $\mathscr{B}$ to respond the queries to $H$ and $H_{1}$, respectively. $\mathscr{B}$ randomly chooses one user $U_{i}^{*}$ and let $b P$ be $U_{i}^{*}$ 's longterm public key. Except $U_{i}^{*}, \mathscr{B}$ computes other users' longterm public/private key pairs by $H . \mathscr{B}$ allows $\mathscr{A}$ to make the following queries.

(i) $H(\cdot)$ : after given $m \in\left\{\left(\right.\right.$ TA's ID $\| U_{i}$ 's ID) $\left.\mid 1 \leq i \leq q\right\}$, $\mathscr{B}$ responds the query, $H(m)$, and maintains the $H$ list as follows.

If $m=$ (TA's ID $\| U_{i}^{*}$ 's ID), $\mathscr{B}$ returns $H(m)=b P$ and stores a record $\left(U_{i}^{*}, b P, \perp, \perp\right)$ in the $H$-list. Otherwise, $\mathscr{B}$ randomly chooses $u_{i} \in \mathbb{Z}_{q}^{*}$, returns $H(m)=u_{i} P$, and stores a record $\left(U_{i}, u_{i} P, u_{i} a P, u_{i}\right)$ in the $H$-list, where $u_{i} P$ and $u_{i} a P$ are the long-term public and private keys, respectively, of $U_{i}$.

(ii) $H_{1}(\cdot)$ : after given $m \in \mathbb{G}_{1}, \mathscr{B}$ randomly chooses $\alpha \epsilon$ $\{0,1\}^{\ell}$, returns $H_{1}(m)=\alpha$, and stores a record $(m, \alpha)$ in the $H_{1}$-list.

(iii) Execute $\left(\Pi_{\mathscr{U}}^{t}\right): \mathscr{A}$ can choose $\mathcal{U}$ and ask $\mathscr{B}$ to run the key agreement protocol. $\mathscr{B}$ returns the public messages $\left(b_{i}, B_{i}, T_{i}, \beta_{i}\right)$ 's of all $U_{i}$ 's in $\mathcal{U}$ to $\mathscr{A}$. $\mathscr{B}$ produces the messages as follows.

If $U_{i}^{*} \in \mathscr{U}, \mathscr{B}$ picks $k_{i}, z, \alpha \in_{R} \mathbb{Z}_{q}^{*}$ and computes $b_{i}=$ $z P-\alpha b P=(z-\alpha b) P, T_{i}=z a P$, and $\beta_{i}=H_{2}\left(T_{i}, \alpha\right)$. For each of the other $U_{j}$ 's $\in \mathscr{U}, \mathscr{B}$ randomly picks $r_{j}, k_{j} \in \mathbb{Z}_{q}^{*}$ and computes $b_{j}=r_{j}^{-1} P, T_{j}=\alpha u_{j} a P+$ $r_{j}^{-1} a P$, and $\beta_{j}=H_{2}\left(T_{j}, \alpha\right)$. Thus, $\mathscr{B}$ can follow the 
protocol to compute $B_{i}$ for each $U_{j} \in \mathcal{U}$ and sets $\alpha=H_{1}(K)$ where $K=\sum_{U_{j} \in \mathcal{U}} k_{j} P$.

(iv) $\operatorname{Send}\left(\Pi_{\widetilde{U}}^{t}, m\right)$ : if $\mathscr{A}$ actively broadcasts the message of users $\widetilde{\mathcal{U}} \subset \mathcal{U}$ to run the key agreement protocol in session $t, \mathscr{B}$ can produce each $\left(b_{i}, B_{i}, T_{i}, \beta_{i}\right)$ of the other $U_{i} \in \mathscr{U}-\widetilde{\mathscr{U}}$ as that in the Execute query. Once if $\mathcal{U}-\tilde{\mathscr{U}}=\left\{U_{i}^{*}\right\}, \mathscr{B}$ can obtain $K$ from $H_{1}$-list while $\mathscr{A}$ is making the $H_{1}$ query for $\alpha$.

(v) Reveal $\left(\Pi_{\mathscr{U}}^{t}\right): \mathscr{B}$ looks up the $H_{1}$-list to obtain $K$ by checking if $H_{2}\left(T_{i}, \alpha\right)=\beta_{i}$ and returns the session conference key SCK $=H_{3}(K)$.

(vi) $\operatorname{Corrupt}\left(U_{i}\right)$ : if $U_{i}=U_{i}^{*}, \mathscr{B}$ returns $\perp$ and aborts the game. Otherwise, $\mathscr{B}$ searches $U_{i}$ in the $H$-list. If $U_{i}$ is not in the $H$-list, $\mathscr{B}$ calls $H$ to produce and store $\left(U_{i}, u_{i} P, u_{i} a P, u_{i}\right)$ in the $H$-list. Then, $\mathscr{B}$ returns $u_{i} a P$.

If $\mathscr{A}$ impersonates $U_{i}^{*}$ at some point, according to Lemma 12, it must send out $T_{i}=\alpha a Q_{i}^{*}+r_{i}^{-1} a P$ with the probability at least $\varepsilon-1 / 2^{q}$, where $H_{1}(K)=\alpha$. Once $\mathscr{A}$ produces a correct $T_{i}, \mathscr{B}$ replays $\mathscr{A}$ with the same random tape by forking lemma. At this time, $\mathscr{A}$ gets two different hashed values $\alpha$ and $\alpha^{\prime}$ and generates two valid $T_{i}=\alpha a b P+$ $r_{i}^{-1} a P$ and $T_{i}^{\prime}=\alpha^{\prime} a b P+r_{i}^{-1} a P . \mathscr{B}$ can compute $c P=(\alpha-$ $\left.\alpha^{\prime}\right)^{-1}\left(T_{i}-T_{i}^{\prime}\right)=a b P$. Finally, $\mathscr{B}$ outputs $a b P$.

Let $X$ and $Y$ be the set of any possible input messages of the random tape and $H_{1}$, respectively. $\mathscr{A}$ fails in making Corrupt queries with probability $1 /|\mathcal{U}|$. $\mathscr{A}$ guesses the value $\alpha=H_{1}(K)$ without making $H_{1}$ queries with probability $2^{-h}$, where $h$ is the length of $H_{1}$ 's output. Therefore, the probability is $\delta=(1 /|\mathcal{U}|)(1-1 /|\mathcal{U}|)\left(1-1 / 2^{h}\right)\left(\varepsilon-1 / 2^{q}\right)$. By splitting lemma, we set $\rho=\delta / 2$ such that $\delta-\rho=\delta-(\delta / 2)=\delta / 2$. Overall, $\mathscr{B}$ performs two executions of $\mathscr{A}$, so that we have

$$
\begin{aligned}
& \varepsilon^{\prime} \geq\left(\frac{\delta}{2}\right)^{2} \\
& =\left(\frac{(1 /|\mathcal{U}|)(1-1 /|\mathcal{U}|)\left(1-1 / 2^{h}\right)\left(\varepsilon-1 / 2^{q}\right)}{2}\right)^{2}, \\
& t^{\prime} \approx 2\left(t+q_{S} \mathcal{O}\left(t_{S}\right)+q_{C} \mathcal{O}\left(t_{C}\right)+q_{R} \mathcal{O}\left(t_{R}\right)\right. \\
& \left.+q_{H} \mathcal{O}\left(t_{H}\right)+q_{H_{1}} \mathcal{O}\left(t_{H_{1}}\right)\right)+\mathcal{O}\left(n^{2}\right) .
\end{aligned}
$$

As for the proof of the interdomain case, $\left(a P, a P^{\prime}, b P\right)$, the parameters of the variant- $\mathrm{CDH}$ problem are the public keys of some TA and some user who belongs to the CA, respectively. The private keys of the other TAs and users are randomly generated by $\mathscr{B}$. Likewise, the proposed interdomain key agreement scheme can also withstand keycompromise impersonation.

6.4. Forward Secrecy. After given broadcast messages, session keys, and all users' long-term keys according to an $\mathscr{I} \mathscr{D} \mathscr{C} \mathscr{K}$ scheme, an adversary makes a Test query and then receives a random string or a session key. The adversary can continue making queries. If no adversary can decide whether the received string is a session key or not with nonnegligible advantage, we say that the $\mathscr{I} \mathscr{D} \mathscr{C} \mathscr{K}$ scheme satisfies forward secrecy.

Definition 14 (forward secrecy). An $\mathscr{I} \mathscr{D} \mathscr{C} \mathscr{K}$ scheme has forward secrecy if any adversary who obtains the other session keys and all users' long term keys can distinguish a previous session key from a random string with the probability at least $(\varepsilon+1 / 2)$ where $\varepsilon$, called the advantage, is negligible.

After running time at most $t$, making at most $q_{H_{1}}$ queries to $H_{1}, q_{E}$ Execute queries, $q_{S}$ Send queries, $q_{C}$ Corrupt queries, and $q_{R}$ Reveal queries, an adversary $\mathscr{A}$ can obtain all users' long-term private keys and receives a string through a Test query. $\mathscr{A}\left(t, q_{E}, q_{S}, q_{C}, q_{R}, q_{H_{1}}, \varepsilon\right)$-FS-breaks our $\mathscr{I} \mathscr{D} \mathscr{C} \mathscr{K}$ scheme if he can determine whether the received string is a previous session key negotiated by users with nonnegligible advantage at least $\varepsilon$.

Theorem 15. If an adversary $\mathscr{A}$ can $\left(t, q_{E}, q_{S}, q_{C}, q_{R}, q_{H_{1}}, \varepsilon\right)$ FS-determine whether SCK is a previous session key or not, where $t$ is the running time, $q_{E}, q_{S}, q_{C}, q_{R}$, and $q_{H_{1}}$ are the numbers of making Send queries, Corrupt queries, Reveal queries, and hash queries to $H_{1}$, respectively, there exists an algorithm to solve the $n-D L D H$ problem with nonnegligible advantage at least $\varepsilon^{\prime}$ in time $t^{\prime}$, where

$$
\begin{aligned}
\varepsilon^{\prime} \geq & \frac{\varepsilon}{q_{0}} \\
t^{\prime} \approx & t+q_{E} \mathcal{O}\left(t_{E}\right)+q_{S} \mathcal{O}\left(t_{S}\right)+q_{C} \mathcal{O}\left(t_{C}\right) \\
& +q_{R} \mathcal{O}\left(t_{R}\right)+q_{H_{1}} \mathcal{O}\left(t_{H_{1}}\right)+\mathcal{O}\left(n^{2}\right),
\end{aligned}
$$

and $q_{0}=q_{E}+q_{S}+q_{C}+q_{R}+q_{H_{1}}$.

Proof. The simulation and the queries answered by $\mathscr{B}$ are the same as those in the proof of Theorem 8 except that $\mathscr{A}$ is allowed to make Corrupt $\left(U_{i}\right)$ query, where $U_{i} \in \mathcal{U}^{*}$, after making Test $\left(\Pi_{U^{*}}^{\ell}\right)$. That is, $\mathscr{A}$ has to activate the $\ell$ th session first before any $U_{i} \in \mathscr{U}^{*}$ is corrupted. In the end, $\mathscr{B}$ can decide whether $Z=\left(x_{n+1}+x_{n+2}+\cdots+x_{2 n}\right) P$ or not according to the value of $b$ output by $\mathscr{A}$, where

$$
\begin{aligned}
\mathcal{E}^{\prime} \geq & \frac{\varepsilon}{q_{0}} \\
t^{\prime} \approx & t+q_{E} \mathcal{O}\left(t_{E}\right)+q_{S} \mathcal{O}\left(t_{S}\right)+q_{C} \mathcal{O}\left(t_{C}\right) \\
& +q_{R} \mathcal{O}\left(t_{R}\right)+q_{H_{1}} \mathcal{O}\left(t_{H_{1}}\right)+\mathcal{O}\left(n^{2}\right) .
\end{aligned}
$$

Similarly, the proposed interdomain key agreement scheme can also achieve forward secrecy.

6.5. Key Control. An adversary who is one of the users can obtain broadcast messages, session keys, and the other users' long-term keys. Then, the adversary is given a preselected 
value. If the adversary cannot make the given preselected value become a new session key with nonnegligible probability, the $\mathscr{I} \mathscr{D} \mathscr{C} \mathscr{K}$ scheme can withstand key control attacks.

Definition 16 (key control). An $\mathscr{I} \mathscr{D} \mathscr{C} \mathscr{K}$ scheme can withstand key control attacks if no adversary can predict a session key or preselect a session key with nonnegligible probability.

Theorem 17. If an adversary $\mathscr{A}$ can $\left(t, q_{E}, q_{S}, q_{C}, q_{R}, q_{H}, \varepsilon\right)$ predict a session key or preselect a session key with probability at least $\varepsilon$, where $t$ is the running time, $q_{E}, q_{S}, q_{C}, q_{R}$, and $q_{H}$ are the numbers of making Send queries, Corrupt queries, Reveal queries, and hash queries to $H$, respectively, there exists an algorithm to solve the $\mathrm{CDH}$ problem with nonnegligible probability at least $\varepsilon^{\prime}$ in time $t^{\prime}$, where

$$
\begin{aligned}
\varepsilon^{\prime} \geq & \frac{\varepsilon}{q_{0}} \\
t^{\prime} \approx & t+q_{E} \mathcal{O}\left(t_{E}\right)+q_{S} \mathcal{O}\left(t_{S}\right)+q_{C} \mathcal{O}\left(t_{C}\right) \\
& +q_{R} \mathcal{O}\left(t_{R}\right)+q_{H} \mathcal{O}\left(t_{H}\right)+\mathcal{O}\left(n^{2}\right),
\end{aligned}
$$

and $q_{0}=q_{E}+q_{S}+q_{C}+q_{R}+q_{H}$.

Proof. At first, $\mathscr{B}$ inputs $\kappa$ to generate pairing parameters and a CDH triple $(P, a P, b P) \in \mathbb{G}_{1}$, where $a, b \in_{R} \mathbb{Z}_{q}^{*}$. We show that an algorithm $\mathscr{B}$ can solve the $\mathrm{CDH}$ problem with the help of an adversary $\mathscr{A} . \mathscr{B}$ 's task is to compute and output the value $c P=a b P$.

$\mathscr{B}$ simulates the system as follows. We define that $\left(s P, s P^{\prime}\right)$ is the system master public key by randomly choosing the master private key $s$. $\mathscr{B}$ allows $\mathscr{A}$ to make the following queries.

(i) $H(\cdot)$ : after given $m \in\left\{\left(\right.\right.$ TA's ID $\| U_{i}$ 's ID) $\left.\mid 1 \leq i \leq q\right\}$, $\mathscr{B}$ responds the query $H(m)$ and maintains the $H$ list as follows: $\mathscr{B}$ randomly chooses $u_{i} \in \mathbb{Z}_{q}^{*}$, returns $H(m)=u_{i} P$, and stores a record $\left(U_{i}, u_{i} P, u_{i} s P, u_{i}\right)$ in the $H$-list.

(ii) $\operatorname{Execute}\left(\Pi_{\mathcal{U}}^{t}\right): \mathscr{A}$ can choose a set $\mathcal{U}$ of users to run the key agreement protocol in session $t$. $\mathscr{B}$ follows the protocol to produce every $\left(b_{i}, B_{i}, T_{i}, \beta_{i}, k_{i}\right)$ and computes $K=\sum_{U_{i} \in \mathcal{U}} k_{i} P$. Finally, $\mathscr{B}$ responds every $\left(b_{i}, B_{i}, T_{i}, \beta_{i}\right)$ to $\mathscr{A}$.

(iii) $\operatorname{Send}\left(\Pi_{\widetilde{U}}^{t}, m\right)$ : if $\mathscr{A}$ can actively send the message of users $\widetilde{\mathcal{U}} \subset \mathcal{U}$ to run the key agreement protocol in session $t, \mathscr{B}$ can produce $\left(b_{i}, B_{i}, T_{i}, \beta_{i}\right)$ of each $U_{i} \in$ $\mathcal{U}-\widetilde{\mathscr{U}}$ as that in the Execute query.

(iv) Reveal $\left(\Pi_{\mathcal{U}}^{t}\right): \mathscr{B}$ returns the session conference key SCK $=H_{3}(K)$.

(v) $\operatorname{Corrupt}\left(U_{i}\right): \mathscr{B}$ searches $U_{i}$ in $H$-list and returns $u_{i} s P$. If $U_{i}$ is not in $H$-list, $\mathscr{B}$ calls $H$ oracle to produce $\left(U_{i}, u_{i} P, u_{i} s P, u_{i}\right)$ and returns $u_{i} s P$.

When $\mathscr{A}$ finishes making the above queries, $\mathscr{B}$ sets $K=x P+b P$, where $x \in_{R} \mathbb{Z}_{q}^{*}$, and returns it to $\mathscr{A}$. Suppose that $\mathscr{A}$ is user $U_{i}$ and $\mathscr{B}$ simulates other users $U_{1}, U_{2}, \ldots, \ldots, U_{i-1}, U_{i+1}, \ldots, U_{n}$ to negotiate a session conference key with $\mathscr{A}$. $\mathscr{B}$ returns $\left\{\left(b_{1}, B_{1}, T_{1}, \beta_{1}\right), \ldots,\left(b_{i-1}, B_{i-1}, T_{i-1}, \beta_{i-1}\right),\left(b_{i+1}, B_{i+1}, T_{i+1}\right.\right.$, $\left.\left.\beta_{i+1}\right), \ldots,\left(b_{n}, B_{n}, T_{n}, \beta_{n}\right)\right\}$ to $\mathscr{A}$, where $x=k_{1}+\cdots+$ $k_{i-1}+k_{i+1}+\cdots+k_{n}$ and $b_{1}=a P$. A must broadcast $B_{i}=\left(k_{i} b_{1}, \ldots, k_{i} b_{i-1}, k_{i} b_{i+1}, \ldots, k_{i} b_{n}\right)$ in round 2 such that $\left(k_{1}+k_{2}+\cdots+k_{n}\right) P=(x+b) P$. Therefore, $k_{i} P=b P$ and $k_{i} b_{1}=a b P$.

Overall, we have that

$$
\begin{aligned}
\mathcal{E}^{\prime} \geq & \frac{\varepsilon}{q_{0}} \\
t^{\prime} \approx & t+q_{E} \mathcal{O}\left(t_{E}\right)+q_{S} \mathcal{O}\left(t_{S}\right)+q_{C} \mathcal{O}\left(t_{C}\right) \\
& \quad+q_{R} \mathcal{O}\left(t_{R}\right)+q_{H} \mathcal{O}\left(t_{H}\right)+\mathcal{O}\left(n^{2}\right) .
\end{aligned}
$$

Following a proof similar to that for the above theorem, we can show that the proposed interdomain key agreement scheme withstands key control attacks.

\section{Discussions}

In this section, we compare our schemes with the schemes of [15-19, 24-27] according to the properties shown in Table 1 and performance factors in Table 2. We prove that our scheme in single domain satisfies all security attributes in Section 6 and it can be easily extended to an interdomain version. Besides, we do our best to extend each scheme in $[15-19,24-27]$ to an interdomain one, but only the schemes $[17,18,24,25]$ can achieve this goal with little modification. Therefore, we use the straightforward way [38] to extend the remaining schemes $[15,16,19,26,27]$ to interdomain versions for performance comparisons. The result is shown in Table 3.

In the design of a conference key agreement scheme, the other researchers always divide the protocol into two main parts: the authentication stage and the stage of the construction of a common secret value. These proposed schemes [15-19, 24-26] all perform the authentication stage first and then make a session conference key, so that they have some problems in security. We have showed that [15$19,24-26]$ are insecure in Section 3 because an adversary $\mathscr{A}$ can get the valid parameters in the authentication stage and replay them in different sessions. In particular, we design our protocol in inverse order such that users share a common secret value first and then run the authentication stage. If we only consider a single TA, some schemes are more efficient than our proposed scheme. However, none of them are secure.

\section{Conclusion}

Many researchers proposed two- or three-party identitybased authenticated key agreement schemes, but general multiparty authenticated key agreement schemes are rare. When the number of the members is more than three, the multiparty scheme is difficult to achieve both security and efficiency at the same time. In this paper, we proposed a novel 
TABle 1: Properties.

\begin{tabular}{|c|c|c|c|c|c|c|c|c|c|}
\hline & {$[24]$} & {$[18]$} & {$[15,16]$} & {$[17]$} & {$[19]$} & [25] & [27] & {$[26]$} & Ours \\
\hline Know session key security & YES & YES & YES & $\mathrm{NO}$ & YES & YES & YES & YES & YES \\
\hline (Perfect) forward secrecy & YES & YES & $\Delta$ & $\mathrm{NO}$ & $\Delta$ & YES & YES & $\Delta$ & YES \\
\hline $\begin{array}{l}\text { No key-compromise } \\
\text { impersonation }\end{array}$ & $\mathrm{NO}$ & NO & NO & YES & NO & $\mathrm{NO}$ & YES & $\mathrm{NO}$ & YES \\
\hline No key control & YES & YES & YES & YES & YES & YES & YES & YES & YES \\
\hline
\end{tabular}

$\Delta$ : partial forward secrecy.

TABLE 2: Performance comparisons in single domain.

\begin{tabular}{|c|c|c|c|c|c|c|c|c|c|c|}
\hline Communication cost & {$[24]$} & {$[18]$} & {$[15,16]$} & [17] & {$[19]$} & {$[25]$} & {$[27]$} & [26] (1) & [26] (2) & Ours \\
\hline Round & 2 & 2 & 2 & 1 & 1 & 3 & 3 & 1 & 2 & 3 \\
\hline Bandwidth & 3 & 3 & 3 & 3 & $(n-1)$ & 4 & $(n-1)$ & $(n+1)$ & 3 & $(n+1)$ \\
\hline Computation cost & {$[24]$} & {$[18]$} & {$[15,16]$} & {$[17]$} & {$[19]$} & {$[25]$} & {$[27]$} & [26] (1) & [26] (2) & Ours \\
\hline Point addition & 9 & 6 & $3 n-3$ & $2 n-3$ & $n-1$ & $2 n+3$ & $n+1$ & - & $n-2$ & $4 n-2$ \\
\hline $\begin{array}{l}\text { Scalar multiplication } \\
\left(\mathbb{G}_{1}, \mathbb{G}_{2}\right)\end{array}$ & 8 & 4 & $n+5$ & $n+3$ & $n$ & $2 n+6$ & $3 n-1$ & - & 2 & $4 n+3$ \\
\hline Scalar multiplication $\left(\mathbb{G}_{T}\right)$ & $2 n$ & - & $n-1$ & $2 n-2$ & - & - & - & $2 n-2$ & 2 & - \\
\hline Pairing & 4 & 4 & 4 & $2 n+1$ & 1 & $n+5$ & $4 n-4$ & $2 n-2$ & 4 & 2 \\
\hline Exponentiation & - & - & $n-2$ & - & - & - & - & - & - & - \\
\hline $\begin{array}{l}\text { Increment/decrement of } \\
\text { computation time } \\
n=100\end{array}$ & $\downarrow 22 \%$ & $\downarrow 19 \%$ & $\downarrow 78 \%$ & $\downarrow 98 \%$ & $\uparrow 48 \%$ & $\downarrow 97 \%$ & $\downarrow 196 \%$ & $\downarrow 98 \%$ & $\downarrow 19 \%$ & - \\
\hline
\end{tabular}

Bandwidth: the total number of messages (points) sent by a user.

$n$ : the number of users.

From [28], 1 exponentiation $\approx 240$ scalar multiplications, 1 point addition $\approx 0.12$ scalar multiplication, 1 scalar multiplication in $\mathbb{G}_{1} \approx 29$ scalar multiplications, 1 scalar multiplication in $\mathbb{G}_{2} \approx 1$ scalar multiplication, and 1 pairing $\approx 10$ exponentiations.

efficient identity-based conference key agreement scheme and proved its security via formal method. Furthermore, our scheme can be extended to an interdomain one.

Consider the application of key agreement among the employees in a company. The focus of the past papers is that how users interact under a single TA. It means that the employees of a company can negotiate a common session key when they want to organize a private conference. But now, our schemes provide more flexibility for the users. Even if the users register with different TAs, they can also negotiate a common session key easily. In other words, when two or more companies want to hold a conference, the employees from different companies can still compute a common session key by our interdomain key agreement scheme.

Our conference key agreement can be applied to the ad hoc networks, too. In the wireless environment, reliable communication and authentication is desired. By performing our method, it is unnecessary for ad hoc sensors to store a large amount of data in advance and they can still negotiate a session key under mutual authentication. We can ensure that the transmitted messages are reliable and also secure against malicious sensors in wireless networks.

\section{Appendices}

\section{A. Shi et al.'s Scheme [19]}

In [19], KGC gives each user, $U_{i}, Q_{i}=\left(I_{i} s_{1}+s_{2}\right) P$ as a public key and $S_{i}=\left(I_{i} s_{1}+s_{2}\right)^{-1} P$ as a private key, where $I_{i}=H\left(\mathrm{ID}_{i}\right)$ is the hashed value of the identity information $\mathrm{ID}_{i} ; s_{1}$ and $s_{2}$ are randomly chosen by KGC. Each $U_{i}(1 \leq i \leq n)$ computes the key $K$ as follows.

Step 1. $U_{i}$ randomly chooses $a_{i}$, computes $T_{i, j}=a_{i} Q_{j}(1 \leq$ $j \leq n, j \neq i$ ), and then sends $T_{i, j}$ to $U_{j}$ for each $j$.

Step 2. After receiving $T_{1, i}, T_{2, i}, \ldots, T_{i-1, i}, T_{i+1, i}, \ldots, T_{n, i}$ from the other users, $U_{i}$ computes

$$
\begin{aligned}
K & =e\left(T_{1, i}+T_{2, i}+\cdots+T_{i-1, i}+a_{i} Q_{i}+T_{i+1, i}+\cdots+T_{n, i}, S_{i}\right) \\
& =e\left(Q_{i}, S_{i}\right)^{a_{1}+a_{2}+\cdots+a_{n}} \\
& =e(P, P)^{a_{1}+a_{2}+\cdots+a_{n}} .
\end{aligned}
$$

There are two security problems shown as follows. 


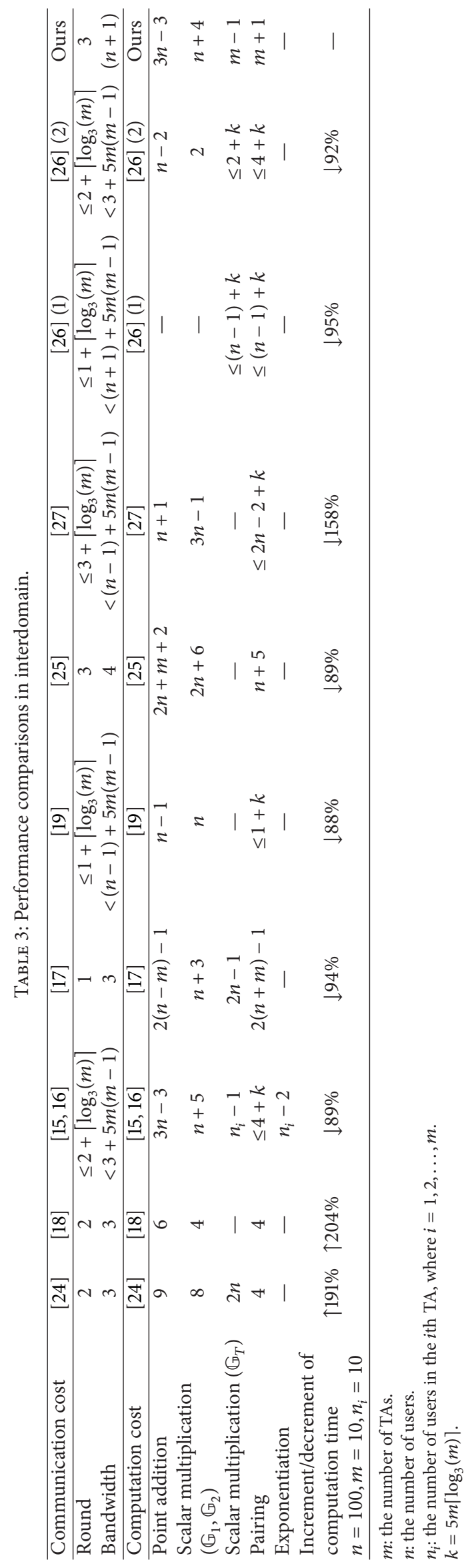




\section{A.1. Key-Compromise Impersonation}

Case 1. Let $U_{1}, U_{2}, \ldots, U_{i}, \ldots, U_{t}, \ldots$, and $U_{n}$ be legal users who are going to negotiate a session conference key. Assume that the private key $S_{i}$ of $U_{i}$ is compromised. Thus, an adversary can act as $U_{i}$ and impersonate another user $U_{t}$. The attack holds even if $U_{i}$ has no $U_{t}$ 's long-term private key $S_{t} . U_{i}$ picks two random integers $a_{i}, a_{t} \in \mathbb{Z}_{q}^{*}$ as her/his ephemeral private keys and then computes $T_{i, j}=a_{i} Q_{j}(1 \leq j \leq n, j \neq i)$ and $T_{t, \hat{j}}=a_{t} Q_{\hat{j}}(1 \leq \widehat{j} \leq n, \widehat{j} \neq t)$. The session conference key $K=e(P, P)^{\left(a_{1}+a_{2}+\cdots+a_{i}+\cdots+a_{t}+\cdots+a_{n}\right)}$ and $U_{t}$ is impersonated by $U_{i}$ in this session [26] and even showed that $U_{i}$ can impersonate $U_{t}$ without participating in session key negotiation.

Case 2. Suppose that an adversary $\mathscr{A}$ obtains $U_{i}$ 's and $U_{t}$ 's long-term private keys $S_{i}$ and $S_{t}$, where $i<t$. $\mathscr{A}$ can impersonate the other users $U_{1}, U_{2}, \ldots, U_{i-1}, U_{i+1}, \ldots, U_{t}, \ldots$, and $U_{n}$ to beguile $U_{i}$ into negotiating a session conference key. $\mathscr{A}$ randomly picks integers $a_{1}, a_{2}, \ldots, a_{i-1}, a_{i+1}, \ldots, a_{n} \in \mathbb{Z}_{q}^{*}$ and computes $T_{j, i}=a_{j} Q_{i}(1 \leq j \leq n, j \neq i) \cdot U_{i}$ randomly picks an integer $a_{i} \in \mathbb{Z}_{q}^{*}$ and computes $T_{i, j}=a_{i} Q_{j}(1 \leq j \leq$ $n, j \neq i)$. Following the protocol, $U_{i}$ can compute the session conference key $K_{i}=e\left(a_{i} Q_{i}+\sum_{j=1, j \neq i}^{n} T_{j, i}, S_{i}\right)=e(P, P)^{z}$, where $z=a_{1}+a_{2}+\cdots+a_{i}+\cdots+a_{n}$. According to the message $T_{i, t}=$ $a_{i} Q_{t}, \mathscr{A}$ computes $e\left(T_{i, t}, S_{t}\right)=e\left(a_{i}\left(I_{t} s_{1}+s_{2}\right) P,\left(I_{t} s_{1}+s_{2}\right)^{-1} P\right)=$ $e(P, P)^{a_{i}}$. Hence, $\mathscr{A}$ can compute the session conference key $K=K_{E}=e(P, P)^{a_{i}} \cdot e(P, P)^{a_{1}+a_{2}+\cdots+a_{i-1}+a_{i+1}+\cdots+a_{n}}=K_{i}$.

A.2. Forward Secrecy. Suppose that an adversary $\mathscr{A}$ gets user $U_{i}$ 's long-term private key $S_{i}$. $\mathscr{A}$ can compute $e\left(\sum_{j=1, j \neq i}^{n} T_{j, i}, S_{i}\right)=e(P, P)^{\sum_{j=1, j \neq i}^{n} a_{j}}$ but cannot compute $e(P, P)^{a_{i}}$. If two or more users' long-term private keys are compromised, $\mathscr{A}$ can compute the previous session keys. Assume that user $U_{t}$ 's long-term private key $S_{t}$ is also obtained by $\mathscr{A}$. $\mathscr{A}$ can compute $e\left(T_{i, t}, S_{t}\right)=e\left(a_{i}\left(I_{t} s_{1}+s_{2}\right) P,\left(I_{t} s_{1}+s_{2}\right)^{-1} P\right)=e(P, P)^{a_{i}}$ and derive the session key $e(P, P)^{a_{1}+a_{2}+\cdots+a_{n}}$. Therefore, this scheme offers partial forward secrecy only.

\section{B. Du et al.'s Scheme $[15,16]$}

Let $\mathrm{ID}_{1}, \mathrm{ID}_{2}, \ldots, \mathrm{ID}_{n}$ be the identifiers of the users $U_{1}, U_{2}, \ldots, U_{n}$. Each $U_{i}(1 \leq i \leq n)$ has the public key $Q_{i}=H_{1}\left(\mathrm{ID}_{i}\right)$ and the private key $S_{i}=s Q_{i}$, where $s$ is the master key of KGC. The protocol is described below.

Step 1. $U_{i}$ randomly chooses $N_{i}$, computes $z_{i}=N_{i} P$ and $T_{i}=H\left(z_{i}\right) S_{i}+N_{i} P_{\text {pub }}$, where $P_{\text {pub }}=s P$, and then broadcasts $\left(z_{i}, T_{i}\right)$.

Step2. If $e\left(\sum_{j=1, j \neq i}^{n} T_{j}, P\right)=e\left(\sum_{j=1, j \neq i}^{n}\left(H\left(z_{j}\right) Q_{j}+z_{j}\right), P_{\text {pub }}\right), U_{i}$ broadcasts $X_{i}=e\left(P_{\text {pub }}, N_{i}\left(z_{i+1}-z_{i-1}\right)\right)$. Besides, let $z_{n+1}=z_{1}$ and $z_{0}=z_{n}$.

Step 3. Finally, $U_{i}$ computes the session key $K=$ $e\left(P_{\text {pub }}, n N_{i} z_{i-1}\right) \cdot X_{i}^{n-1} \cdot X_{i+1}^{n-2} \cdots X_{n}^{i-1} \cdot X_{1}^{i-2} \cdot X_{2}^{i-3} \cdots X_{i-2}=$ $e(P, P)^{\left(N_{1} N_{2}+N_{2} N_{3}+\cdots+N_{n-1} N_{n}+N_{n} N_{1}\right) s}$.

The following two attacks are valid.
B.1. Key-Compromise Impersonation [29]. When $U_{i}$ wants to negotiate a session conference key with others, she/he must compute the authenticated factor $\left(z_{i}, T_{i}\right)$ and broadcast it. Two adversaries $\mathscr{A}_{1}$ and $\mathscr{A}_{2}$ can get $\left(z_{i}, T_{i}\right)$ in a previous session. Suppose that $\mathscr{A}_{1}$ is $U_{i-1}$ and $\mathscr{A}_{2}$ is $U_{i+1}$. Thus, $\mathscr{A}_{1}$ and $\mathscr{A}_{2}$ can impersonate $U_{i}$ by rebroadcasting $\left(z_{i}, T_{i}\right)$ that satisfies the verification formula in Step 2 . When $\mathscr{A}_{1}$ and $\mathscr{A}_{2}$ collude, they can compute $X_{i}=e\left(z_{i},\left(N_{i+1}-N_{i-1}\right) P_{\text {pub }}\right)=$ $e\left(N_{i} P, s\left(z_{i+1}-z_{i-1}\right)\right)=e\left(P_{\text {pub }}, N_{i}\left(z_{i+1}-z_{i-1}\right)\right)$.

Now, $\mathscr{A}_{1}$ and $\mathscr{A}_{2}$ have a valid message $\left(z_{i}, T_{i}, X_{i}\right)$, so that they can impersonate $U_{i}$ to construct a session conference key without being detected by other users.

B.2. Forward Secrecy. Assume that an adversary $\mathscr{A}$ gets TA's long-term private key s. $U_{1}, U_{2}, \ldots$, and $U_{n}$ negotiated a key $K$ in a previous session and $\mathscr{A}$ got all transmitted messages $\left(z_{1}, T_{1}, X_{1}\right),\left(z_{2}, T_{2}, X_{2}\right), \ldots$, and $\left(z_{n}, T_{n}, X_{n}\right)$. Then $\mathscr{A}$ can compute the previous session key $K$ as follows:

$$
\begin{aligned}
K & =e\left(z_{i}, s n z_{i-1}\right) \cdot X_{i}^{n-1} \cdot X_{i+1}^{n-2} \cdots X_{i-2} \\
& =e\left(P_{\mathrm{pub}}, n N_{i} z_{i-1}\right) \cdot X_{i}^{n-1} \cdot X_{i+1}^{n-2} \cdots X_{i-2} .
\end{aligned}
$$

Du et al. improved their scheme in [16]. They added a synchronous counter $c$ which was held by all users. The initial value of $c$ is 1 and $c$ is increased by 1 after a successful session. In the improved scheme, they modified $T_{i}=H\left(z_{i}\right)$ $c S_{i}+N_{i} P_{\text {pub }}$ in Step 1 and $U_{i}$ verified if $e\left(\sum_{j=1, j \neq i}^{N} T_{j}, P\right)=$ $e\left(\sum_{j=1, j \neq i}^{N}\left(H\left(z_{j}\right) c Q_{j}+z_{j}\right), P_{\text {pub }}\right)$ in Step 2. However, [16] still has the same vulnerability as [15] to the key-compromise impersonation attack (because $\mathscr{A}_{1}$ and $\mathscr{A}_{2}$ who act as $U_{i-1}$ and $U_{i+1}$, resp., know $c$, which is public among users) and lacks forward secrecy as shown above.

\section{Zhang et al.'s Scheme [18]}

Each $U_{i}(1 \leq i \leq n)$ has the public key $Q_{i}=H_{1}\left(\mathrm{ID}_{i}\right)$ and the private key $S_{i}=s Q_{i}$, which are the same as those of Du et al's scheme. The protocol is shown below.

Step 1. $U_{i}$ randomly chooses $N_{i}$ and computes $z_{i}=N_{i} P$ and $T_{i}=H\left(z_{i}\right) S_{i}+N_{i} P_{\text {pub }}$ and then sends $z_{i}$ with the signature $T_{i}$ to $U_{i-2}, U_{i-1}, U_{i+1}$, and $U_{i+2}$.

Step 2. After verifying each received $z_{i}$ by checking that $e\left(T_{i}, P\right)=e\left(\left(H\left(z_{i}\right) Q_{i}+z_{i}\right), P_{\text {pub }}\right), U_{1}$ and $U_{n}$ compute $Y_{1 R}=e\left(z_{2}, z_{3}\right)^{N_{1}}, Y_{1 L}=e\left(z_{n}, z_{2}\right)^{N_{1}}=e\left(z_{1}, z_{2}\right)^{N_{n}}=Y_{n R}$, $Y_{n L}=e\left(z_{n-2}, z_{n-1}\right)^{N_{n}}$ if $n$ is odd; otherwise $U_{1}$ and $U_{n-1}$ compute $Y_{1 R}=e\left(z_{2}, z_{3}\right)^{N_{1}}, Y_{1 L}=e\left(z_{n-1}, z_{n}\right)^{N_{1}}=e\left(z_{n}\right.$, $\left.z_{1}\right)^{N_{n-1}}=Y_{(n-1) R}, Y_{(n-1) L}=e\left(z_{n-3}, z_{n-2}\right)^{N_{n-1}}$. The other $U_{i}$ 's compute $Y_{i L}=e\left(z_{i-2}, z_{i-1}\right)^{N_{i}}$ and $Y_{i R}=e\left(z_{i+1}, z_{i+2}\right)^{N_{i}}$ if $i$ is odd; otherwise they do nothing. Finally, each $U_{i}$, with an odd $i$, broadcasts $X_{i}=H\left(Y_{i L}\right) \oplus H\left(Y_{i R}\right)$ with the signature.

Step 3. Each user $U_{i}$ ( $i$ is odd) and $U_{i+1}$ can compute the session conference key $K=H\left(Y_{1 L}\right)+H\left(Y_{3 L}\right)+\cdots+H\left(Y_{n L}\right)$ if $n$ is odd where $H\left(Y_{j L}\right)=H\left(Y_{i R}\right) \oplus X_{i} \oplus X_{i-2} \oplus \cdots \oplus X_{j}$ 
(when $i \geq j$ ) or $H\left(Y_{j L}\right)=H\left(Y_{i R}\right) \oplus X_{i} \oplus X_{i-2} \oplus \cdots \oplus X_{1} \oplus$ $X_{n} \oplus X_{n-2} \oplus \cdots \oplus X_{j}$ (when $i<j$ ) for each odd $j$. Otherwise, $K=H\left(Y_{1 L}\right)+H\left(Y_{3 L}\right)+\cdots+H\left(Y_{(n-1) L}\right)$ if $n$ is even where $H\left(Y_{j L}\right)=H\left(Y_{i R}\right) \oplus X_{i} \oplus X_{i-2} \oplus \cdots \oplus X_{j}$ (when $i \geq j$ ) or $H\left(Y_{j L}\right)=H\left(Y_{i R}\right) \oplus X_{i} \oplus X_{i-2} \oplus \cdots \oplus X_{1} \oplus X_{n-1} \oplus X_{n-3} \oplus \cdots \oplus X_{j}$ (when $i<j$ ) for each odd $j$.

It is easy to impersonate a user whose index is even in the scheme since the users only sends the message $\left(z_{i}, T_{i}\right)$. We show an example as follows. Suppose that there are six users $U_{1}, U_{2}, U_{3}, U_{4}, U_{5}$, and $U_{6}$ who want to negotiate a conference key. By this scheme, we can know that $U_{4}$ only submits $\left(z_{4}, T_{4}\right)$ to $U_{2}, U_{3}, U_{5}$, and $U_{6}$ in Step 1. If $U_{2}, U_{3}, U_{5}$, and $U_{6}$ collude, they can negotiate a randomly-chosen pair of $\left(z_{4}^{\prime}, T_{4}^{\prime}\right)$ to cheat $U_{1}$ without $U_{4}$ joining in the session because $U_{1}$ does not need to verify $\left(z_{4}^{\prime}, T_{4}^{\prime}\right)$. Besides, even though $U_{i}$ broadcasts the message $\left(z_{i}, T_{i}\right)$ and every user can check the correctness of the message, the scheme still suffers from keycompromise impersonation by resending $\left(z_{i}, T_{i}\right)$ as that of $\mathrm{Du}$ et al.'s scheme [29].

\section{Kim et al.'s Scheme [17]}

First, KGC sets up $s$ as the master key and $\left(P_{\text {pub }}, P\right)$ as public parameters, where $P_{\text {pub }}=s P$. Then, KGC generates a key pair for each user. Let $\mathrm{ID}_{1}, \mathrm{ID}_{2}, \ldots, \mathrm{ID}_{n}$ be the identifiers of users $U_{1}, U_{2}, \ldots, U_{n}$, respectively. Each $U_{i}(1 \leq i \leq n)$ has the public key $Q_{i}=H_{1}\left(\mathrm{ID}_{i}\right)$ and the private key $S_{i}=s Q_{i}$.

Step 1. $U_{i}$ broadcasts $a_{i} P_{\text {pub }}$ with the signature $\left(P_{i}, T_{i}\right)$, where $P_{i}=a P, T_{i}=H\left(P_{i}, a_{i} P_{\text {pub }}\right) S_{i}+a a_{i} P_{\text {pub }}, a_{i}$ and $a$ are randomly chosen by $U_{i}$.

Step 2. After verifying the signature by checking that $e\left(T_{j}, P\right)=e\left(H\left(P_{j}, a_{j} P_{\text {pub }}\right) Q_{j}, P_{\text {pub }}\right) \cdot e\left(a_{j} P_{\text {pub }}, P_{j}\right)$, the conference key $K_{s}$ is computed as follows: $K_{s}=H_{2}(K)$ where $K=\prod_{i=1}^{N} e\left(Q_{i}, a_{i} P_{\text {pub }}\right)$.

This scheme has a serious problem. In the protocol, since every user $U_{i}$ broadcasts the message $\left(a_{i} P_{\text {pub }}, P_{i}, T_{i}\right)$, one can collect all $a_{i} P_{\text {pub }}$ 's and compute all $Q_{i}$ 's to derive $K=$ $\prod_{i=1}^{N} e\left(Q_{i}, a_{i} P_{\mathrm{pub}}\right)$ even if she/he does not join the session.

\section{E. Choi et al.'s Scheme [24]}

First, KGC sets up $s$ as the master key and $\left(P_{\text {pub }}, P\right)$ as public parameters, where $P_{\text {pub }}=s P$. Then, KGC generates a key pair for each user. Let $\mathrm{ID}_{1}, \mathrm{ID}_{2}, \ldots, \mathrm{ID}_{n}$ be the identifiers of users $U_{1}, U_{2}, \ldots, U_{n}$, respectively. Each $U_{i}(1 \leq i \leq n)$ has the public key $Q_{i}=H_{1}\left(\mathrm{ID}_{i}\right)$ and the private key $S_{i}=s Q_{i}$.

Step 1. $U_{i}$ broadcasts the signature $\left(P_{i}, T_{i}\right)$, where $P_{i}=a_{i} P$, $T_{i}=H\left(P_{i}\right) S_{i}+a_{i} P_{\text {pub }}, a_{i}$ is randomly chosen by $U_{i}$.

Step 2. After receiving $\left(P_{i-1}, T_{i-1}\right),\left(P_{i+1}, T_{i+1}\right)$, and $\left(P_{i+2}, T_{i+2}\right)$, $U_{i}$ verifies the messages by checking whether $e\left(T_{i-1}+T_{i+1}+\right.$ $\left.T_{i+2}, P\right)=e\left(P_{i-1}+P_{i+1}+P_{i+2}+H\left(P_{i-1}\right) Q_{i-1}+H\left(P_{i+1}\right) Q_{i+1}+\right.$ $\left.H\left(P_{i+2}\right) Q_{i+2}, P_{\text {pub }}\right) . U_{i}$ then broadcasts $D_{i}=e\left(a_{i}\left(P_{i+2}-\right.\right.$ $\left.\left.P_{i-1}\right), P_{i+1}\right)$.
Step 3. $U_{i}$ can compute the session key $K_{i}=e\left(a_{i} P_{i-1}, P_{i+1}\right)^{n}$ $D_{i}^{n-1} D_{i+1}^{n-2} \cdots D_{n}^{i-1} D_{1}^{i-2} D_{2}^{i-3} \cdots D_{i-2}$.

Reference [30] has shown that [24] is vulnerable to keycompromise impersonation as follows.

Suppose that attacker $\mathscr{A}_{1}$ is $U_{i-1}$ and attacker $\mathscr{A}_{2}$ is $U_{i+2}$. Thus, $\mathscr{A}_{1}$ and $\mathscr{A}_{2}$ can rebroadcast $\left(P_{i}, T_{i}\right)$ and then broadcast $D_{i}=e\left(P_{i}, P_{i+1}\right)^{-a_{i-1}+a_{i+2}}=e\left(a_{i}\left(P_{i+2}-P_{i-1}\right), P_{i+1}\right)$ to impersonate $U_{i}$.

\section{F. Zhou et al.'s Schemes [26]}

Zhou et al. proposed two group key agreement schemes. One is a one-round scheme with security proofs. The other based on the former is a two-round scheme with lower communication cost. These schemes are depicted as below:

KGC sets up $s$ as the master key and $\left(P_{\text {pub }}, P\right)$ as public parameters, where $P_{\text {pub }}=s P$. Then, KGC generates a key pair for each user. Let $\mathrm{ID}_{1}, \mathrm{ID}_{2}, \ldots, \mathrm{ID}_{n}$ be the identifiers of users $U_{1}, U_{2}, \ldots, U_{n}$, respectively. Each $U_{i}(1 \leq i \leq n)$ has the public key $Q_{i}=H_{1}\left(\operatorname{ID}_{i}\right)$ and the private key $S_{i}=s Q_{i}$.

In the former scheme, each user $U_{i}$ randomly picks $\delta_{i}$, $r_{i}$, and $k_{i}$, computes $P_{i}^{j}=H_{2}\left(e\left(S_{i}, Q_{j}\right) \cdot \delta_{i}\right) \oplus r_{i}$ for each $1 \leq j \leq n$ and $j \neq i$, and broadcasts $D_{i}=$ $\left(\delta_{i}, P_{i}^{1}, \ldots, P_{i}^{i-1}, P_{i}^{i+1}, \ldots, P_{i}^{n}, H_{3}\left(r_{i}\right) \oplus k_{i}\right)$. After receiving all $D_{j}=\left(R_{j}, P_{j}^{1}, \ldots, P_{j}^{j-1}, P_{j}^{j+1}, \ldots, P_{j}^{n}, V_{j}\right)^{\prime} \mathrm{s}, U_{i}$ computes $k_{j}^{\prime}=$ $H_{3}\left(H_{2}\left(e\left(Q_{j}, S_{i}\right) \cdot R_{j}\right) \oplus P_{j}^{i}\right) \oplus V_{j}$ for each $1 \leq j \leq n$ and $j \neq i$. Thus, the session key $K_{i}=k_{1}^{\prime} \oplus k_{2}^{\prime} \oplus \cdots \oplus k_{i-1}^{\prime} \oplus k_{i} \oplus k_{i+1}^{\prime} \oplus \cdots \oplus k_{n}^{\prime}$.

In the latter scheme, only $U_{1}$ randomly picks $\delta, r$, and $k_{1}$ and broadcasts $D_{1}=\left(R, P_{2}, \ldots P_{n}, V, W\right)$ such that $D_{1}=\left(\delta, r \oplus H_{4}\left(e\left(S_{1}, Q_{2}\right) \cdot \delta\right), \ldots, r \oplus H_{4}\left(e\left(S_{1}, Q_{n}\right) \cdot \delta\right)\right.$, $\left.H_{5}(r) k_{1} P, k_{1} P_{\text {pub }}\right)$. When each $U_{i}(2 \leq i \leq n)$ receives $D_{1}$, he computes $r^{\prime}=H_{4}\left(e\left(Q_{1}, S_{i}\right) \cdot R\right) \oplus P_{i}=r$ and broadcasts $D_{i}=$ $\left(X_{i}, Y_{i}\right)=\left(H_{5}(r) k_{i} P, k_{i} P_{\text {pub }}\right)$, where $k_{i}$ is randomly chosen by $U_{i}$. Finally, all $U_{i}$ 's $(1 \leq i \leq n)$ compute $z_{1}=H_{5}(r)^{-1} V$ and $z_{j}=H_{5}(r)^{-1} X_{j}, 2 \leq j \leq n$, and verify $z_{1}, \ldots, z_{n}$ by checking $e\left(P, \sum_{j=2}^{n} Y_{j}+W\right)=e\left(P_{\text {pub }}, \sum_{j=1}^{n} z_{j}\right)$. Finally, all users can compute the session key $K_{i}=H_{6}\left(z_{1}\right) \oplus \cdots \oplus H_{6}\left(z_{n}\right)$.

Both of the schemes only achieve partial forward secrecy. Once all of the private keys $S_{i}$ 's of the users are revealed or KGC's secret key $s$ is corrupted, an attacker is able to compute previous session keys by intercepting the previous broadcast messages. Moreover, the schemes cannot withstand key-compromise impersonation. In the first scheme, if the private keys $S_{1}, \ldots, S_{i-1}, S_{i+1}, \ldots S_{n}$ are revealed, an attacker $\mathscr{A}$ can impersonate $U_{i}$ by broadcasting the message $D_{i}$ with random strings $\left\{\delta_{i}, r_{i}, k_{i}\right\}$ and $P_{i}^{j}=H_{2}\left(e\left(S_{j}, Q_{i}\right) \cdot \delta_{i}\right) \oplus r_{i}$. In the second scheme, if $U_{1}$ 's private key $S_{1}$ is revealed, an attacker $\mathscr{A}$ can impersonate any other $U_{i}$ by computing and broadcasting $D_{i}=\left(H_{5}(r) k_{i} P, k_{i} P_{\text {pub }}\right), 2 \leq i \leq n$, where $\delta, r, k_{1}$, and $k_{i}$ are randomly chosen.

\section{G. Yao et al.'s Scheme [25]}

KGC sets up $s$ as the master key and $(R, P)$ as public parameters, where $R=s P$. Then, KGC generates a key pair for each user. Let $\mathrm{ID}_{1}, \mathrm{ID}_{2}, \ldots, \mathrm{ID}_{n}$ be the identifiers of users 
$U_{1}, U_{2}, \ldots, U_{n}$, respectively. Each $U_{i}(1 \leq i \leq n)$ has the public key $Q_{i}=H_{0}\left(\operatorname{ID}_{i}\right)$ and the private key $S_{i}=s Q_{i}$.

Step 1. Each $U_{i}$ generates a random string $r_{i}$, computes $E_{i}=r_{i} P$ and $F_{i}=H\left(U, e\left(E_{i}, R\right)\right) S_{i}+r_{i} R$, where $U=\mathrm{ID}_{1}\left\|\mathrm{ID}_{2}\right\| \cdots \| \mathrm{ID}_{n}$, and broadcasts $\left(E_{i}, F_{i}\right)$.

Step 2. Each $U_{i}$ verifies the received $\left(E_{j}, F_{j}\right), 1 \leq j \leq n$ and $j \neq i$, by checking whether $e\left(\sum_{j \neq i} F_{j}, P\right)=e\left(\sum_{j \neq i} H\left(U, e\left(E_{j}\right.\right.\right.$, $\left.R)) Q_{j}+E_{j}, R\right)$. If true, $U_{i}$ computes and broadcasts $Y_{i}=r_{i} T$ and $X_{i}=r_{i}\left(E_{i+1}-E_{i-1}+T\right)$, where $T=H_{0}\left(\mathrm{ID}_{1}\left\|E_{1}\right\| \cdots\left\|\mathrm{ID}_{n}\right\| E_{n}\right)$.

Step 3. After receiving $\left(X_{j}, Y_{j}\right)^{\prime}$ s, $U_{i}$ checks if $e\left(\sum_{j \neq i} Y_{j}, P\right)=e\left(\sum_{j \neq i} E_{j}, T\right)$. Then, $U_{i}$ computes $Z_{i}=e\left(n r_{i} E_{i-1}+\sum_{j=0}^{n-1}(n-1-j)\left(X_{i+j}-Y_{i+j}\right), R\right)$ and broadcasts $C_{i}=H\left(i\|U\| E_{1}\|\cdots\| E_{n}\left\|X_{1}\right\| \cdots\left\|X_{n}\right\| Y_{1}\|\cdots\| Y_{n} \| Z_{i}\right)$.

Step 4. $U_{i}$ checks whether each received $C_{j}$ equals $H\left(j\|U\| E_{1}\|\cdots\| E_{n}\left\|X_{1}\right\| \cdots\left\|X_{n}\right\| Y_{1}\|\cdots\| Y_{n} \| Z_{i}\right), \quad$ where $Z_{i}=Z_{j}$. If the condition holds, the session key $K_{i}=$ $H\left(U\left\|E_{1}\right\| \cdots\left\|E_{n}\right\| X_{1}\|\cdots\| X_{n}\left\|Y_{1}\right\| \cdots\left\|Y_{n}\right\| Z_{i}\left\|C_{1}\right\| \cdots \| C_{n}\right)$.

The scheme is not immune to key-compromise impersonation, either. Suppose that an adversary $\mathscr{A}$ who obtains $U_{i}$ 's private key $S_{i}$ attempts to impersonate $U_{t}$. $\mathscr{A}$ can rebroadcast previous messages $\left(E_{t}^{\prime}, F_{t}^{\prime}\right)$ and $\left(X_{t}^{\prime}, Y_{t}^{\prime}\right)$ in Step 1 and Step 2, respectively. Then, $\mathscr{A}$ broadcasts $C_{t}=$ $H\left(t\|U\| E_{1}\|\cdots\| E_{n}\left\|X_{1}\right\| \cdots\left\|X_{n}\right\| Y_{1}\|\cdots\| Y_{n} \| Z_{i}\right)$ in Step 3.

\section{Conflict of Interests}

The authors declare that there is no conflict of interests regarding the publication of this paper.

\section{Acknowledgment}

This work was partially supported by the Ministry of Science and Technology of Taiwan under Grant MOST 103-2221-E110-057, NSYSU-KMU Joint Research Project (NSYSUKMU 103-I001), and Aim for the Top University Plan of the National Sun Yat-sen University and Ministry of Education, Taiwan.

\section{References}

[1] W. Diffie and M. E. Hellman, "New directions in cryptography," IEEE Transactions on Information Theory, vol. 22, no. 6, pp. 644-654, 1976.

[2] R. Dutta and R. Barua, "Overview of key agreement protocols," Tech. Rep. 2005/289, Cryptology ePrint Archive, 2005, http://eprint.iacr.org/2005/289.pdf.

[3] A. Shamir, "Identity-based cryptosystems and signature schemes," in Advance in Cryptology, vol. 196 of Lecture Notes in Computer Science, pp. 47-53, Springer, Berlin, Germany, 1985.

[4] D. Boneh and M. Franklin, "Identity-based encryption from the Weil pairing," SIAM Journal on Computing, vol. 32, no. 3, pp. 586-615, 2003.

[5] G. Frey, M. Müller, and H. Rüuck, "The Tate pairing and the discrete logarithm applied to elliptic curve cryptosystems," IEEE
Transactions on Information Theory, vol. 45, no. 5, pp. 1717-1719, 1999.

[6] A. Joux, "A one round protocol for tripartite Diffie-Hellman," Journal of Cryptology, vol. 17, no. 4, pp. 263-276, 2004.

[7] N. P. Smart, "Identity-based authenticated key agreement protocol based on Weil pairing," Electronics Letters, vol. 38, no. 13, pp. 630-632, 2002.

[8] L. Chen, Z. Cheng, and N. P. Smart, "Identity-Based Key Agreement Protocols from Pairings," Cryptology, Report 2006/199, 2006, http://eprint.iacr.org/2006/199.pdf.

[9] L. Chen and C. Kudla, "Identity based key agreement protocols from pairings," in Proceedings of the 16th IEEE Computer Security Foundations Workshop, pp. 219-233, Pacific Grove, Calif, USA, 2003.

[10] Y. J. Choie, E. Jeong, and E. Lee, "Efficient identity-based authenticated key agreement protocol from pairings," Applied Mathematics and Computation, vol. 162, no. 1, pp. 179-188, 2005.

[11] N. McCullagh and P. S. L. M. Barreto, "A new two-party identitybased authenticated key agreement," in Topics in CryptologyCT-RSA 2005, vol. 3376 of Lecture Notes in Computer Science, pp. 262-274, Springer, Berlin, Germany, 2005.

[12] M. Bellare and P. Rogaway, "Entity authentication and key distribution," in Advances in Cryptology-CRYPTO 93, vol. 773 of Lecture Notes in Computer Science, pp. 232-249, Springer, 1994.

[13] S. S. Al-Riyami and K. G. Paterson, "Tripartite authenticated key agreement protocols from pairings," in Cryptography and Coding, vol. 2898 of Lecture Notes in Computer Science, pp. 332359, Springer, 2003.

[14] K. Shim and S. S. Woo, "Cryptanalysis of tripartite and multiparty authenticated key agreement protocols," Information Sciences, vol. 177, no. 4, pp. 1143-1151, 2007.

[15] X. Du, Y. Wang, J. Ge, and Y. Wang, "An ID-based authenticated two round multi-party key agreement," Cryptology ePrint Archive, Report 2003/247, 2003, http://eprint.iacr.org/2003/247 .pdf.

[16] X. Du, Y. Wang, J. Ge, and Y. Wang, "An improved ID-based authenticated group key agreement scheme," Cryptology ePrint Archive, Report 2003/260, 2003, http://eprint.iacr.org/2003/ 260.pdf.

[17] J. S. Kim, H. C. Kim, K. J. Ha, and K. Y. Yoo, "One round identity-based authenticated conference key agreement protocol," in Proceedings of the 3rd European Conference (ECUMN '04), vol. 3262 of Lecture Notes in Computer Science, pp. 407416, 2004.

[18] P. Zhang, C. Ye, X. Li, and X. Ma, "An efficient keys agreement for multi-party's communication," in Proceedings of the 6th International Conference on Parallel and Distributed Computing, Applications and Technologies (PDCAT '05), pp. 267-269, Dalian, China, December 2005.

[19] Y. Shi, G. Chen, and J. Li, "ID-based one round authenticated group key agreement protocol with bilinear pairings," in Proceeding of the International Conference on Information Technology: Coding and Computing (ITCC '05), vol. 1, pp. 757761, April 2005.

[20] I. F. Blake, G. Seroussi, and N. P. Smart, Advances in Elliptic Curve Cryptography, London Mathematical Society Lecture Note Series, No. 317, 2005.

[21] F. Bao, R. H. Deng, and H. Zhu, "Variations of Diffie-Hellman problem," in Infromation and Communications Security, vol. 2836 of Lecture Notes in Computer Science, pp. 301-312, 2003. 
[22] D. Boneh, X. Boyen, and H. Shacham, "Short group signatures," in Advances in Cryptology-CRYPTO 04, vol. 3152 of Lecture Notes in Computer Science, pp. 41-55, Springer, 2004.

[23] X. Boyen and B. Waters, "Anonymous hierarchical identitybased encryption (without random oracles)," in Advances in Cryptology-CRYPTO 2006, vol. 4117 of Lecture Notes in Computer Science, pp. 209-307, Springer, Berlin, Germany, 2006.

[24] K. Y. Choi, J. Y. Hwang, and D. H. Lee, "Efficient IDbased group key agreement with bilinear maps," in Public Key Cryptography-PKC 2004, vol. 2947 of Lecture Notes in Computer Science, pp. 130-144, Springer, Berlin, Germany, 2004.

[25] G. Yao, H. Wang, and Q. Jiang, "An authenticated 3-round identity-based group key agreement protocol," in Proceedings of the 3rd International Conference on Availability, Security, and Reliability (ARES '08), pp. 538-543, Barcelona, Spain, March 2008.

[26] L. Zhou, W. Susilo, and Y. Mu, "Efficient ID-based authenticated group key agreement from bilinear pairings," in Proceedings of the 2nd International Conference (MSN '06), vol. 4325 of Lecture Notes in Computer Science, pp. 521-532, 2006.

[27] W. Yuan, L. Hu, H. Li, and J. Chu, "An enhanced authenticated 3round identity-based group key agreement protocol," in Green Communications and Networks, vol. 113 of Lecture Notes in Electrical Engineering, pp. 549-556, Springer, Amsterdam, The Netherlands, 2012.

[28] N. Koblitz, A. Menezes, and S. Vanstone, "The state of elliptic curve cryptography," Designs, Codes and Cryptography, vol. 19, no. 2-3, pp. 173-193, 2000.

[29] F. G. Zhang and X. F. Chen, "Attack on Two ID-based Authenticated Group Key Agreement Schemes," Cryptology, Report 2003/259.

[30] F. Zhang and X. Chen, "Attack on an ID-based authenticated group key agreement scheme from PKC 2004," Information Processing Letters, vol. 91, no. 4, pp. 191-193, 2004.

[31] H. Park, T. Asano, and K. Kim, "Improved ID-based authenticated Group key agreement secure against impersonation attack by insider," in Proceedings of the Symposium on Cryptography and Information Security, Otsu, Japan, 2009.

[32] V. Shoup, "Lower bounds for discrete logarithms and related problems," in Advances in Cryptology-EUROCRYPT '97, vol. 1233 of Lecture Notes in Computer Science, pp. 256-266, 1997.

[33] M. Bellare and P. Rogaway, "Random oracles are practical: a paradigm for designing efficient protocols," in Proceedings of the 1st ACM Conference on Computer and Communications Security, pp. 62-73, November 1993.

[34] S. Blake-Wilson, D. Johnson, and A. Menezes, "Key agreement protocols and their security analysis," in Crytography and Coding: Proceedings of the 6th IMA International Conference on Cryptography and Coding, vol. 1355 of Lecture Notes in Computer Science, pp. 30-45, 1997.

[35] D. Pointcheval and J. Stern, "Security proofs for signature schemes," in Advances in Cryptology-EUROCRYPT '96, vol. 1070 of Lecture Notes in Computer Science, pp. 387-398, Springer, Berlin, Germany, 1996.

[36] D. Pointcheval and J. Stern, "Security arguments for digital signatures and blind signatures," Journal of Cryptology, vol. 13, no. 3, pp. 361-396, 2000.

[37] M. Bellare, J. A. Garary, and T. Rabin, "Fast batch verification for modular exponentiation and digital signatures," in Advances in Cryptology-EUROCRYPT'98, vol. 1403 of Lecture Notes in
Computer Science, pp. 236-250, Springer, Berlin, Germany, 1998.

[38] R. Barua, R. Dutta, and P. Sarkar, "Extending Joux's protocol to multi party key agreement," in Progress in CryptologyINDOCRYPT 03, vol. 2904 of Lecture Notes in Computer Science, pp. 205-217, 2003. 


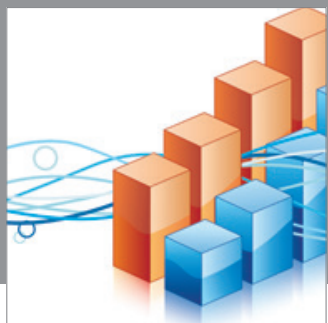

Advances in

Operations Research

mansans

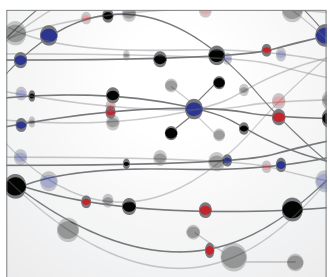

The Scientific World Journal
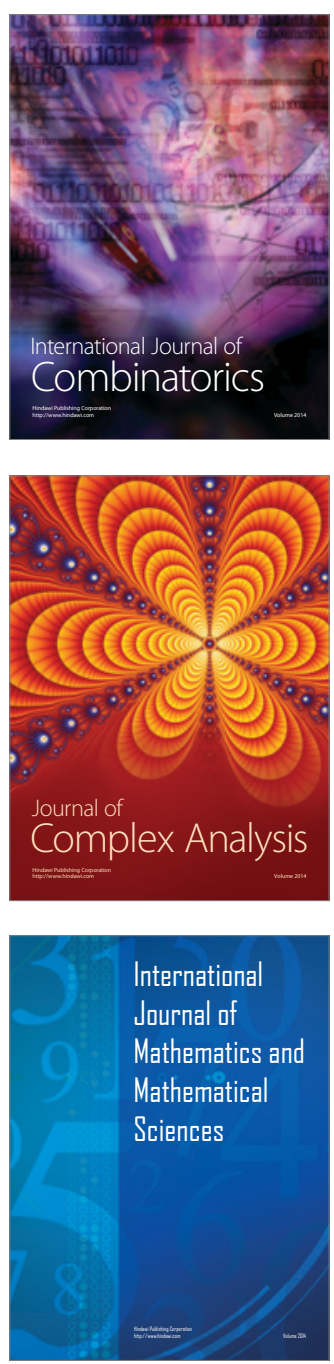
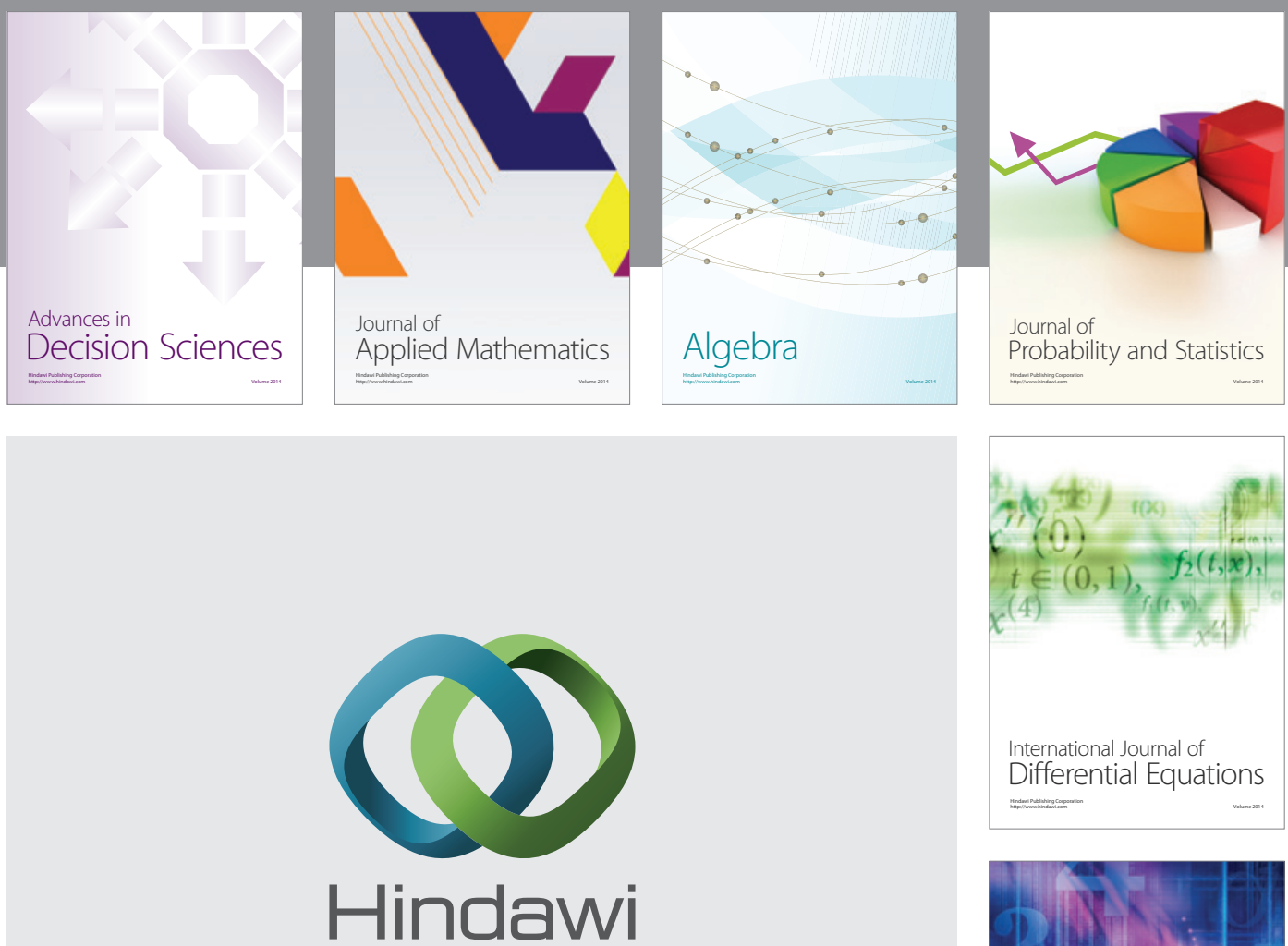

Submit your manuscripts at http://www.hindawi.com
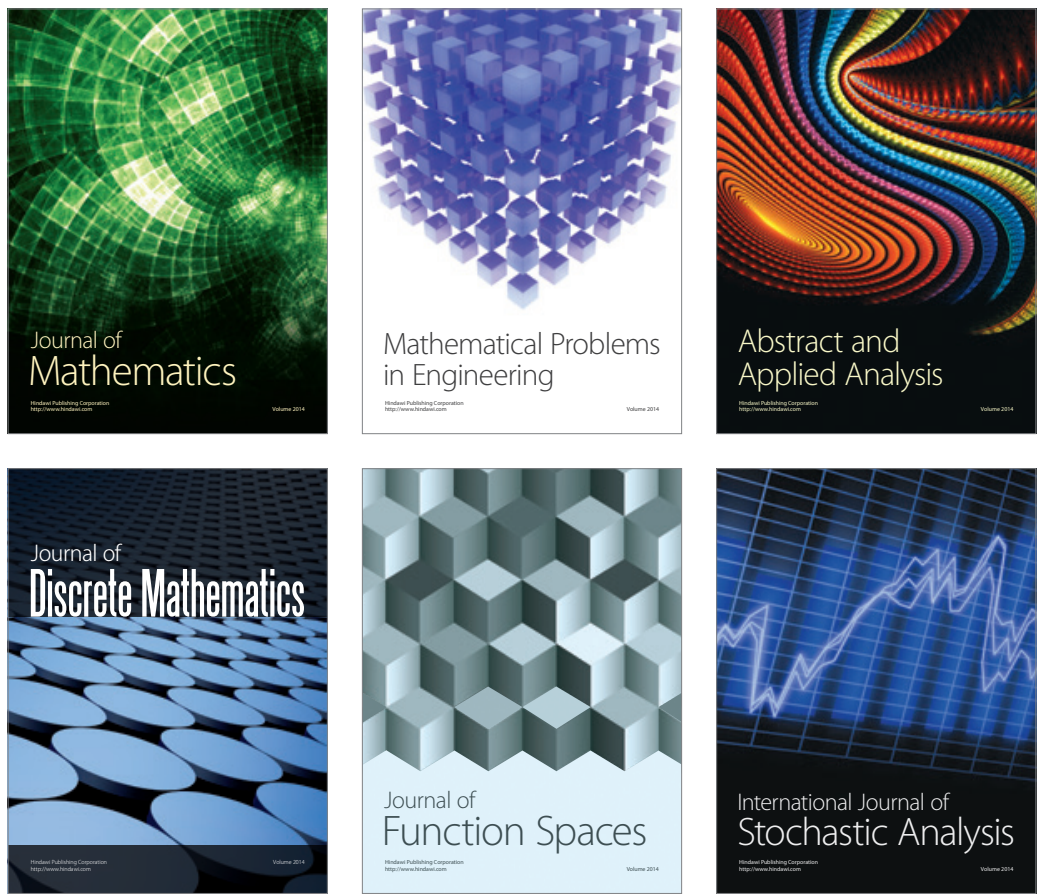

Journal of

Function Spaces

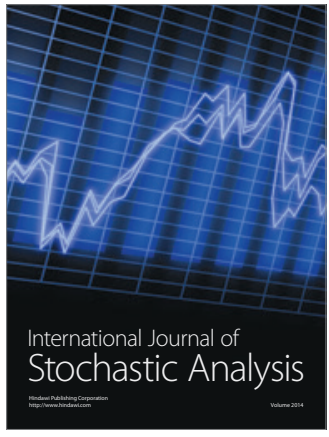

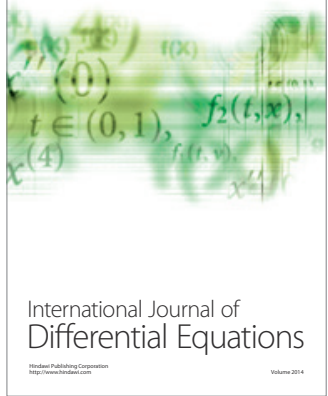
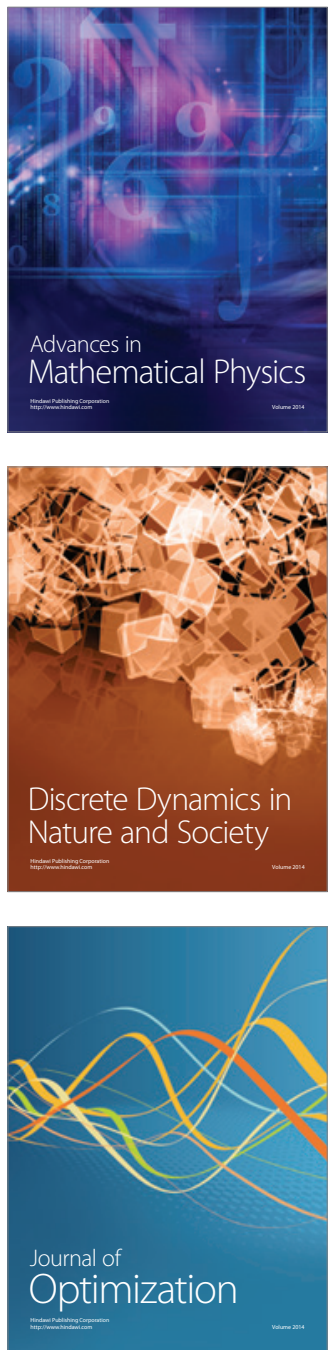